\title{
Thirty Years of the International Journal of Intelligent Systems: A Bibliometric Review
}

\author{
José M. Merigó, ${ }^{1,2, *}$ Fabio Blanco-Mesa, ${ }^{3, \dagger}$ Anna M. Gil-Lafuente, ${ }^{4}$ \\ Ronald R. Yager ${ }^{5}$ \\ ${ }^{1}$ Department of Management Control and Information Systems, School of Eco- \\ nomics and Business, University of Chile, 8330015 Santiago, Chile \\ ${ }^{2}$ King Saud University, Riyadh, Saudi Arabia \\ ${ }^{3}$ Department of Business Administration, Antonio Nariño University, Tunja, \\ Colombia \\ ${ }^{4}$ Department of Business Administration, University of Barcelona, 08034 \\ Barcelona, Spain \\ ${ }^{5}$ Machine Intelligence Institute, lona College, New Rochelle, NY 10801, USA
}

The International Journal of Intelligent Systems was created in 1986. Today, the journal is 30 years old. To celebrate this anniversary, this study develops a bibliometric review of all of the papers published in the journal between 1986 and 2015. The results are largely based on the Web of Science Core Collection, which classifies leading bibliographic material by using several indicators including total number of publications and citations, the $h$-index, cites per paper, and citing articles. The work also uses the VOS viewer software for visualizing the main results through bibliographic coupling and co-citation. The results show a general overview of leading trends that have influenced the journal in terms of highly cited papers, authors, journals, universities and countries. (c) 2016 Wiley Periodicals, Inc.

\section{INTRODUCTION}

The International Journal of Intelligent Systems (IJIS) is a leading journal in the field of computer science and artificial intelligence with a focus on theories based on intelligent systems construction. It was created in 1986 by the current editor-inchief, Ronald R. Yager. Over time, the journal has grown significantly and today is a main reference worldwide. ${ }^{1}$ It is included in the Journal Citation Reports of the Web of Science Core Collection, which only indexes those journals that are recognized as being of the highest quality. Currently, it is a monthly journal published by John Wiley \& Sons, international publisher.

*Author to whom all correspondence should be addressed; e-mail: jmerigo@ fen.uchile.cl.

${ }^{\dagger}$ Visiting distinguished scientist. 
Bibliometrics is a research field that studies bibliographic material quantitatively ${ }^{2}$ by analyzing a research area and identifying its leading trends. Bibliometrics can be developed in a wide range of contexts including the analysis of a research topic,,$^{3,4}$ journals,,${ }^{5,6}$ institutions, ${ }^{7}$ and countries. ${ }^{8}$ In computer science, there are many bibliometric studies concerning a wide range of issues including journals ${ }^{9,10}$ and countries. ${ }^{11,13}$ More specifically, there are several bibliometric studies close to the scope of IJIS including computational intelligence, ${ }^{14}$ fuzzy sets and systems, ${ }^{1}$ aggregation operators, ${ }^{15}$ intuitionistic fuzzy sets,${ }^{16}$ linguistic decision making, ${ }^{17}$ gray systems, ${ }^{18}$ and ant colony optimization. ${ }^{19}$

In 2016, IJIS celebrates its 30th anniversary. Motivated by this event, the aim of this paper is to develop a bibliometric overview of the journal between 1986 and 2015. By doing so, we can see who is leading the journal and the main trends that have had more influence. The analysis considers a wide range of issues including the publication and citation structure of the journal, most-cited papers, the most influential authors, institutions and countries, and citing articles. The study also uses the VOS viewer software ${ }^{20}$ to visualize leading trends in the journal through bibliographic coupling ${ }^{21}$ and co-citation analysis. ${ }^{22}$

The remainder of the paper is structured as follows: Section 2 briefly reviews the bibliometric methods used in the analysis. Section 3 presents the publication and citation structure of the journal. Section 4 develops a mapping of the documents published in the journal by using the VOS viewer software. Section 5 summarizes the main findings and conclusions of the paper.

\section{BIBLIOMETRIC METHODS}

There is a wide range of methodologies that can be implemented when developing a bibliometric review. This study focuses on a general overview that presents different indicators so that each reader can reach his or her own understanding of the bibliometric data. The main reason for this approach is that there is no single method agreed upon by everyone for evaluating research. The key problem is that some people prefer to focus on certain bibliometric indicators, whereas other people might prefer other indicators. However, it is clear that the comparison between productivity and influence can be measured with the number of publications and citations, respectively. Some authors assign more importance to productivity and vice versa. Therefore, each method can evaluate the data differently. An alternative to obtain a unified method could be using a consensual process in which everyone agrees on the importance of each variable. However, this process would be a subjective method because it is largely based on the opinion of some experts in the field.

To provide a complete picture of the data, the work uses total number of publications and citations, citations per paper, the $h$-index, ${ }^{23,24}$ citing articles and citation thresholds. The $h$-index is an index that combines publications with citations. If a variable has an $h$-index of $X$, then there are $X$ papers inside the set of papers considered that have received $X$ citations or more. Moreover, there are not $X+1$ papers with $X+1$ citations or more. The citing articles are those that cite the 
material considered in the analysis. The citation thresholds considered in the study analyze the number of papers that are above a specific number of citations.

The work uses the Web of Science (WoS) database, which is currently owned by Thomson \& Reuters. Specifically, we use the WoS Core Collection, which indexes documents that are usually recognized as having the highest quality. WoS currently has more than 15 thousand journals and more than 50 million documents. Other databases that classify scientific research include Scopus and Google Scholar.

The search process uses the name of the journal "International Journal of Intelligent Systems" with the search option "Publication Name." The search considered all of the documents published in the journal between 1986 and 2015. Note that WoS only includes the journal since 1987 . Therefore, for 1986, we developed a manual search through the Cited Reference Search tool and examined the documents available in 1986 on the webpage of the journal. The search process found 1602 documents published in the journal during its first 30 years. Currently, IJIS has received 16,865 citations and has an $h$-index of 54. That is, of the 1602 documents, 54 have received 54 or more citations. ${ }^{25}$ Note that the search process was performed in November 2015, when all of the documents published in the journal in 2015 were available in WoS.

Finally, note that the study provides a general picture with the aim of identifying the leading trends that have affected the journal during these three decades. However, many limitations can occur due to the particular issues that affect different types of research. For example, WoS always provides one unit to any coauthor of a paper and one unit to each participating institution and country. However, this approach gives an advantage to papers written by many authors because it does not divide the paper according to the number of coauthors or institutions involved in the paper. Therefore, papers by authors who tend to publish individually obtain lower results than do papers with several authors because the effort to write a single-author paper is greater than that for a multiauthored paper. Moreover, WoS does not consider the number of pages of an article or many other related issues that could condition the analysis. In any case, the paper uses a standard methodology that can offer a representative view of the results found in the database.

\section{RESULTS}

\subsection{Publication and Citation Evolution of IJIS}

IJIS started publishing papers in 1986. At that time, it was a quarterly journal. In the nineties, it increased from five issues in 1990 to seven issues in 1991, eight issues in 1992, and nine in 1993. Finally, it consolidated as a monthly journal in 1994. Owing to this pattern, the first years saw an important increase in the annual number of documents published in the journal. Figure 1 shows the evolution of the number of papers published annually.

As seen, the number of papers increases until 2001, when it began stabilizing at approximately 60 to 70 papers per year. Observe that in recent years, the annual number of papers published in the journal has decreased slightly. Owing to the increase of research and journals worldwide, the average citations received in each 


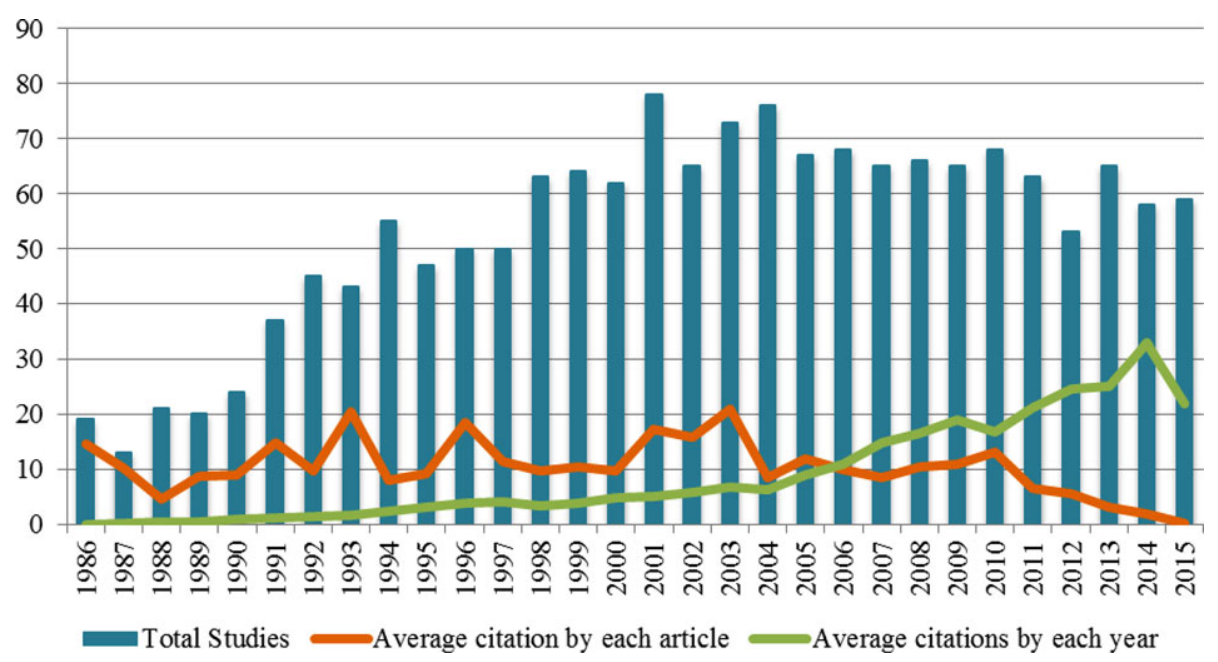

Figure 1. Number of annual documents published in IJIS.

year is increasing over time. However, the average citations by each article are less in recent years because these articles are new in the literature and have not had time to obtain a significant citation rate. Obviously, these data are dynamic and will change in the future once these articles reach maturity.

Next, let us examine the citations that the documents published in IJIS have received over time. Table I presents the citations that the documents published in each year have received in WoS. Note that the citation thresholds indicate the number of papers published in a specific year that have surpassed a number of citations.

Currently, 2001, 2002, and 2003 are the years when the documents published in the journal reached the highest impact, with more than 1000 citations. Note that slightly more than $1 \%$ of the articles have received more than 100 citations, approximately $25 \%$ have received at least 10 citations and $77 \%$ of the articles have received at least one citation.

Another interesting issue concerning citations is to analyze the most-cited papers published in the journal. Table II presents the 50 most-cited papers.

Yager has the most-cited paper in the journal. This paper, published in 1996, analyzed new contributions to the knowledge of the ordered weighted average (OWA) operator. ${ }^{26}$ Zeshui Xu (one with Qingli Da) has the second and third mostcited papers, ${ }^{27,28}$ and Vicenç Torra the fourth and fifth most-cited papers. ${ }^{29,30}$ Note that the paper by Torra published in 2010, in which he introduces the concept of hesitant fuzzy sets, has the highest number of cites per year.

IJIS has received 16,865 citations during these 30 years. Through WoS, we can analyze those papers that cite the journal through the "citing articles" link available in the citation report section. Table III presents those articles that have cited IJIS, classified by journals, years, institutions, countries, and authors. 
Table I. Annual citation structure of IJIS.

\begin{tabular}{|c|c|c|c|c|c|c|c|c|c|c|}
\hline & $\geq 100$ & $\geq 50$ & $\geq 20$ & $\geq 10$ & $\geq 5$ & $\geq 1$ & Total studies & Total citations & $\mathrm{TC}$ & Impact factor \\
\hline 1986 & - & 1 & 6 & 6 & 10 & 15 & 19 & 276 & - & - \\
\hline 1987 & - & - & 2 & 4 & 7 & 10 & 13 & 133 & 2 & - \\
\hline 1988 & - & - & 1 & 3 & 5 & 15 & 21 & 98 & 18 & - \\
\hline 1989 & - & 1 & 2 & 4 & 9 & 16 & 20 & 175 & 19 & - \\
\hline 1990 & - & - & 2 & 4 & 3 & 17 & 24 & 216 & 26 & - \\
\hline 1991 & 1 & 3 & 6 & 14 & 21 & 31 & 37 & 550 & 52 & - \\
\hline 1992 & 1 & 3 & 6 & 12 & 16 & 33 & 45 & 436 & 70 & - \\
\hline 1993 & 2 & 4 & 10 & 16 & 27 & 38 & 43 & 879 & 76 & - \\
\hline 1994 & - & 2 & 6 & 14 & 22 & 42 & 55 & 435 & 130 & - \\
\hline 1995 & - & 2 & 6 & 14 & 18 & 40 & 47 & 436 & 153 & - \\
\hline 1996 & 1 & 3 & 7 & 14 & 20 & 40 & 50 & 921 & 190 & - \\
\hline 1997 & 1 & 1 & 4 & 11 & 20 & 43 & 50 & 568 & 203 & 0.379 \\
\hline 1998 & - & 3 & 10 & 15 & 34 & 55 & 63 & 611 & 215 & 0.354 \\
\hline 1999 & - & 2 & 14 & 18 & 32 & 50 & 64 & 670 & 244 & 0.410 \\
\hline 2000 & - & 1 & 12 & 22 & 30 & 53 & 62 & 597 & 308 & 0.450 \\
\hline 2001 & 3 & 7 & 15 & 30 & 50 & 62 & 78 & 1341 & 393 & 0.398 \\
\hline 2002 & 3 & 3 & 13 & 23 & 39 & 57 & 65 & 1027 & 384 & 0.390 \\
\hline 2003 & 3 & 8 & 16 & 28 & 45 & 67 & 73 & 1521 & 506 & 0.875 \\
\hline 2004 & - & 2 & 8 & 16 & 35 & 61 & 76 & 637 & 483 & 0.603 \\
\hline 2005 & 1 & 2 & 5 & 18 & 33 & 56 & 67 & 792 & 609 & 0.657 \\
\hline 2006 & - & 1 & 9 & 21 & 32 & 54 & 68 & 669 & 743 & 0.429 \\
\hline 2007 & - & 1 & 8 & 21 & 32 & 56 & 65 & 554 & 977 & 0.667 \\
\hline 2008 & - & 4 & 9 & 16 & 33 & 55 & 66 & 692 & 1105 & 0.860 \\
\hline 2009 & 1 & 2 & 9 & 15 & 31 & 57 & 65 & 715 & 1247 & 1.194 \\
\hline 2010 & 2 & 4 & 9 & 19 & 26 & 51 & 68 & 899 & 1169 & 1.331 \\
\hline 2011 & 1 & 2 & 4 & 6 & 18 & 50 & 63 & 416 & 1349 & 1.653 \\
\hline 2012 & - & - & 3 & 9 & 19 & 41 & 53 & 291 & 1313 & 1.416 \\
\hline 2013 & - & - & 1 & 5 & 10 & 42 & 65 & 194 & 1652 & 1.411 \\
\hline 2014 & - & - & 1 & 1 & 4 & 30 & 58 & 102 & 1926 & 1.886 \\
\hline 2015 & - & - & - & - & - & 10 & 59 & 14 & 1305 & N/A \\
\hline Total & 20 & 62 & 204 & 399 & 681 & 1247 & 1602 & 16,865 & 16,865 & \\
\hline Percentage & 1.25 & 3.87 & 12.73 & 24.91 & 42.51 & 77.84 & 100 & & & \\
\hline
\end{tabular}

Source: Own elaboration based on WoS. $\geq:$ it is an accumulated value; TC: total citations received in that year

Information Sciences is the journal that primarily cites journal. IJIS appears in second position. Note that IJIS does not occupy the first position because it publishes a lower number of articles than does Information Sciences. Therefore, it is easier for Information Sciences to cite IJIS more frequently. Most of the leading journals in the field ${ }^{1}$ appear in the Top 30. Concerning years, as seen in Table I, there is a progressive growth over time in the number of citations. The University of Granada has the highest number of citations to the journal. In terms of countries, China is the leading citing country. Concerning authors, Zeshui Xu, Ronald Yager, and Francisco Herrera are the authors that provide the most citations per author to the journal.

\subsection{Leading Authors, Universities, and Countries in the Journal}

Many important contributions have been published in the journal by a wide range of authors, institutions, and countries. In this section, let us focus on those 
Table II. Most-cited papers published in IJIS.

\begin{tabular}{|c|c|c|c|c|c|}
\hline $\mathrm{R}$ & Article & Name of authors & $\mathrm{TC}$ & YP & $\mathrm{C} / \mathrm{Y}$ \\
\hline 1 & $\begin{array}{l}\text { Quantifier guided aggregation using OWA } \\
\text { operators }\end{array}$ & Yager, RR & 438 & 1996 & 23.05 \\
\hline 2 & $\begin{array}{l}\text { An overview of operators for aggregating } \\
\text { information }\end{array}$ & $\mathrm{Xu}, \mathrm{ZS} ; \mathrm{Da}, \mathrm{QL}$ & 360 & 2003 & 30.00 \\
\hline 3 & $\begin{array}{l}\text { An overview of methods for determining OWA } \\
\text { weights }\end{array}$ & $\mathrm{Xu}, \mathrm{ZS}$ & 267 & 2005 & 26.70 \\
\hline 4 & The weighted OWA operator & Torra, V & 267 & 1997 & 14.83 \\
\hline 5 & Hesitant fuzzy sets & Torra, $\mathrm{V}$ & 246 & 2010 & 49.20 \\
\hline 6 & On aggregation operations of linguistic labels & $\begin{array}{l}\text { Delgado, M; Verdegay, } \\
\text { JL; Vila, MA }\end{array}$ & 225 & 1993 & 10.23 \\
\hline 7 & $\begin{array}{l}\text { Information granulation and rough set } \\
\text { approximation }\end{array}$ & Yao, YY & 199 & 2001 & 14.21 \\
\hline 8 & The uncertain OWA operator & $\mathrm{Xu}, \mathrm{ZS} ; \mathrm{Da}, \mathrm{QL}$ & 197 & 2002 & 15.15 \\
\hline 9 & $\begin{array}{l}\text { A review of evolutionary artificial neural } \\
\text { networks }\end{array}$ & Yao, $\mathrm{X}$ & 168 & 1993 & 7.64 \\
\hline 10 & $\begin{array}{l}\text { Generalized aggregation operators for } \\
\text { intuitionistic fuzzy sets }\end{array}$ & $\begin{array}{l}\text { Zhao, H; Xu, ZS; Ni, MF; } \\
\text { Liu, SS }\end{array}$ & 157 & 2010 & 31.40 \\
\hline 11 & $\begin{array}{l}\text { A taxonomy for the crossover operator for } \\
\text { real-coded genetic algorithms: An } \\
\text { experimental study }\end{array}$ & $\begin{array}{l}\text { Herrera, F; Lozano, M; } \\
\text { Sanchez, AM }\end{array}$ & 151 & 2003 & 12.58 \\
\hline 12 & $\begin{array}{l}\text { Comparative study of alternative types of } \\
\text { knowledge reduction in inconsistent systems }\end{array}$ & Kryszkiewicz, M & 144 & 2001 & 10.29 \\
\hline 13 & Linguistic decision-making models & $\begin{array}{l}\text { Delgado, M; Verdegay, } \\
\text { JL; Vila, MA }\end{array}$ & 134 & 1992 & 5.83 \\
\hline 14 & $\begin{array}{l}\text { The ordered weighted geometric averaging } \\
\text { operators }\end{array}$ & $\mathrm{Xu}, \mathrm{ZS} ; \mathrm{Da}, \mathrm{QL}$ & 132 & 2002 & 10.15 \\
\hline 15 & Rough approximation by dominance relations & $\begin{array}{l}\text { Greco, S; Matarazzo, B; } \\
\text { Slowinski, R }\end{array}$ & 128 & 2002 & 9.85 \\
\hline 16 & $\begin{array}{l}\text { Group decision making with incomplete fuzzy } \\
\text { linguistic pref. rel. }\end{array}$ & $\begin{array}{l}\text { Alonso, S; Cabrerizo, FJ; } \\
\text { Chiclana, F; Herrera, F; } \\
\text { Herrera-Viedma, E }\end{array}$ & 115 & 2009 & 19.17 \\
\hline 17 & $\begin{array}{l}\text { A generalized defuzzification method via bad } \\
\text { distributions }\end{array}$ & Filev, DP; Yager, RR & 114 & 1991 & 4.75 \\
\hline 18 & $\begin{array}{l}\text { On distance and correlation measures of hesitant } \\
\text { fuzzy information }\end{array}$ & $\mathrm{Xu}, \mathrm{ZS} ; \mathrm{Xia}, \mathrm{MM}$ & 110 & 2011 & 27.50 \\
\hline 19 & $\begin{array}{l}\text { A consensus-reaching process under } \\
\text { intuitionistic fuzzy preference relations }\end{array}$ & Szmidt, E; Kacprzyk, J & 109 & 2003 & 9.08 \\
\hline 20 & $\begin{array}{l}\text { Information granules: Towards foundations of } \\
\text { granular computing }\end{array}$ & Skowron, A; Stepaniuk, J & 108 & 2001 & 7.71 \\
\hline 21 & $\begin{array}{l}\text { A consistency-based procedure to estimate } \\
\text { missing pairwise preference values }\end{array}$ & $\begin{array}{l}\text { Alonso, S; Chiclana, F; } \\
\text { Herrera, F; } \\
\text { Herrera-Viedma, E; } \\
\text { Alcala-Fdez, J; Porcel, } \\
\text { C }\end{array}$ & 98 & 2008 & 14.00 \\
\hline 22 & $\begin{array}{l}\text { Induced aggregation operators in decision } \\
\text { making with the Dempster-Shafer belief } \\
\text { structure }\end{array}$ & $\begin{array}{l}\text { Merigo, JM; Casanovas, } \\
\quad \mathrm{M}\end{array}$ & 97 & 2009 & 16.17 \\
\hline 23 & Note on ranking fuzzy triangular numbers & $\begin{array}{l}\text { Facchinetti, G; Ricci, RG; } \\
\quad \text { Muzzioli, S }\end{array}$ & 97 & 1998 & 5.71 \\
\hline 24 & $\begin{array}{l}\text { Time and time again: The many ways to } \\
\text { represent time }\end{array}$ & Allen, JF & 95 & 1991 & 3.96 \\
\hline
\end{tabular}


Table II. Continued

\begin{tabular}{|c|c|c|c|c|c|}
\hline $\mathrm{R}$ & Article & Name of authors & $\mathrm{TC}$ & YP & $\mathrm{C} / \mathrm{Y}$ \\
\hline 25 & $\begin{array}{l}\text { Induced ordered weighted geometric operators } \\
\text { and their use in the aggregation of } \\
\text { multiplicative preference relations }\end{array}$ & $\begin{array}{l}\text { Chiclana, F; } \\
\text { Herrera-Viedma, E; } \\
\text { Herrera, F; Alonso, S }\end{array}$ & 87 & 2004 & 7.91 \\
\hline 26 & $\begin{array}{l}\text { Nearest prototype classifier designs: An } \\
\text { experimental study }\end{array}$ & Bezdek, JC; Kuncheva, LI & 87 & 2001 & 6.21 \\
\hline 7 & $\begin{array}{l}\text { Determining the importance weights for the } \\
\text { design requirements in the house of quality } \\
\text { using the fuzzy analytic network approach }\end{array}$ & $\begin{array}{l}\text { Buyukozkan, G; Ertay, T; } \\
\text { Kahraman, C; Ruan, D }\end{array}$ & 86 & 2004 & 7.82 \\
\hline 28 & $\begin{array}{l}\text { Approaches to knowledge reductions in } \\
\text { inconsistent systems }\end{array}$ & $\begin{array}{l}\text { Zhang, WX; Mi, JS; Wu, } \\
\text { WZ }\end{array}$ & 86 & 2003 & 7.17 \\
\hline 29 & $\begin{array}{l}\text { Forecasting enrolments using high-order fuzzy } \\
\text { time series and genetic algorithms }\end{array}$ & Chen, SM; Chung, NY & 85 & 2006 & 9.44 \\
\hline 30 & $\begin{array}{l}\text { Fuzzy neural network with fuzzy signals and } \\
\text { weights }\end{array}$ & $\begin{array}{l}\text { Hayashi, Y; Buckley, JJ; } \\
\text { Czogala, E }\end{array}$ & 83 & 1993 & 3.77 \\
\hline 31 & $\begin{array}{l}\text { An introduction to bipolar representations of } \\
\text { information and preference }\end{array}$ & Dubois, D; Prade, H & 81 & 2008 & 11.57 \\
\hline 32 & $\begin{array}{l}\text { Negation functions based semantics for ordered } \\
\text { linguistic labels }\end{array}$ & Torra, V & 79 & 1996 & 4.16 \\
\hline 3 & $\begin{array}{l}\text { Dominance relation and rules in an incomplete } \\
\text { ordered information system }\end{array}$ & Shao, MW; Zhang, WX & 77 & 2005 & 7.70 \\
\hline 34 & $\begin{array}{l}\text { ypicality, and uncertainty in class } \\
\text { s }\end{array}$ & $\begin{array}{l}\text { Dubois, D; Prade, H; } \\
\quad \text { Rossazza, JP }\end{array}$ & 74 & 1991 & 3.08 \\
\hline 35 & $\begin{array}{l}\text { A study of the origin and uses of the ordered } \\
\text { weighted geometric operator in multicriteria } \\
\text { decision making }\end{array}$ & $\begin{array}{l}\text { Herrera, F; } \\
\text { Herrera-Viedma, E; } \\
\text { Chiclana, F }\end{array}$ & 71 & 2003 & 5.92 \\
\hline 36 & Some comments on interval valued fuzzy sets & $\begin{array}{l}\text { Gehrke, M; Walker, C; } \\
\text { Walker, E }\end{array}$ & 71 & 1996 & 3.74 \\
\hline 37 & $\begin{array}{l}\text { The uncertain induced quasi-arithmetic OWA } \\
\text { operator introduction }\end{array}$ & $\begin{array}{l}\text { Merigo, JM; Casanovas, } \\
\quad \mathrm{M}\end{array}$ & 65 & 2011 & 16.25 \\
\hline 38 & $\begin{array}{l}\text { Constructing deliberative agents with case-based } \\
\text { reasoning technology }\end{array}$ & Corchado, JM; Laza, R & 65 & 2003 & 5.42 \\
\hline 39 & $\begin{array}{l}\text { Rough reduction, in algebra view and } \\
\text { information view }\end{array}$ & Wang, G & 65 & 2003 & 5.42 \\
\hline 40 & $\begin{array}{l}\text { Linguistic aggregation operators of selection } \\
\text { criteria in fuzzy information-retrieval }\end{array}$ & Bordogna, G; Pasi, G & 65 & 1995 & 3.25 \\
\hline & On a class of operators for expert-systems & & 4 & 1993 & 2.91 \\
\hline 42 & $\begin{array}{l}\text { A model of an information retrieval system with } \\
\text { unbalanced fuzzy linguistic information }\end{array}$ & $\begin{array}{l}\text { Herrera-Viedma, E; } \\
\text { Lopez-Herrera, AG }\end{array}$ & 63 & 2007 & 7.88 \\
\hline 43 & $\begin{array}{l}\text { Genetic learning of fuzzy rule-based } \\
\text { classification systems cooperating with fuzzy } \\
\text { reasoning methods }\end{array}$ & $\begin{array}{l}\text { Cordon, O; del Jesus, MJ; } \\
\text { Herrera, F }\end{array}$ & 61 & 1998 & 3.59 \\
\hline 44 & On the concept of immediate probabilities & $\begin{array}{l}\text { Yager, RR; Engemann, } \\
\text { KJ; Filev, DP }\end{array}$ & 59 & 1995 & 2.95 \\
\hline & Evalu & & & & 4 \\
\hline 46 & $\begin{array}{l}\text { Mining fuzzy association rules and fuzzy } \\
\text { frequency episodes for intrusion detection }\end{array}$ & , SM & 8 & 00 & 3.87 \\
\hline 47 & $\begin{array}{l}\text { A fuzzy object-oriented data model for } \\
\text { managing vague and uncertain information }\end{array}$ & $\begin{array}{l}\text { Bordogna, G; Pasi, G; } \\
\quad \text { Lucarella, D }\end{array}$ & 57 & 1999 & 3.56 \\
\hline 48 & $\begin{array}{l}\text { Generalized ordered weighted logarithm } \\
\text { aggregation operators and their applications to } \\
\text { group decision making }\end{array}$ & Zhou, LG; Chen, HY & 56 & 2010 & 11.20 \\
\hline
\end{tabular}

(Continued) 
Table II. Continued

\begin{tabular}{|c|c|c|c|c|c|}
\hline $\mathrm{R}$ & Article & Name of authors & $\mathrm{TC}$ & YP & $\mathrm{C} / \mathrm{Y}$ \\
\hline 49 & $\begin{array}{l}\text { HILK: A new methodology for designing highly } \\
\text { interpretable linguistic knowledge bases using } \\
\text { the fuzzy logic formalism }\end{array}$ & $\begin{array}{l}\text { Alonso, JM; Magdalena, } \\
\text { L; Guillaume, S }\end{array}$ & 56 & 2008 & 8.00 \\
\hline 50 & $\begin{array}{l}\text { Induced Choquet ordered averaging operator } \\
\text { and its application to group decision making }\end{array}$ & Tan, CQ; Chen, XH & 55 & 2010 & 11.00 \\
\hline
\end{tabular}

Source: Own elaboration based on WoS.

Table III. IJIS citing articles classified by journals, years, universities, countries, and authors.

\begin{tabular}{|c|c|c|c|c|c|c|c|c|c|c|}
\hline $\mathrm{R}$ & Journals & $\mathrm{TP}$ & Years & $\mathrm{TP}$ & Universities & TP & Countries & $\mathrm{TP}$ & $\begin{array}{l}\text { Name of } \\
\text { authors }\end{array}$ & $\mathrm{TP}$ \\
\hline 1 & ISC & 448 & 2015 & 764 & U Granada & 457 & $\begin{array}{l}\text { People's } \\
\text { Republic } \\
\text { of China }\end{array}$ & 2844 & $\mathrm{Xu}$ ZS & 184 \\
\hline 2 & IJIS & 427 & 2014 & 1089 & CNRS & 209 & USA & 1514 & Yager RR & 166 \\
\hline 3 & FSS & 396 & 2013 & 1033 & Southeast U China & 170 & Spain & 1441 & Herrera F & 159 \\
\hline 4 & ESA & 285 & 2012 & 837 & Iona College & 163 & UK & 698 & Merigo JM & 107 \\
\hline 5 & IEEETFS & 266 & 2011 & 794 & U Jaen & 159 & Italy & 559 & $\begin{array}{l}\text { Herrera- } \\
\text { Viedma } \\
\text { E }\end{array}$ & 101 \\
\hline 6 & IJAR & 227 & 2010 & 739 & $\begin{array}{l}\text { Polish Academy } \\
\text { of Sciences }\end{array}$ & 145 & Taiwan & 538 & Martinez L & 76 \\
\hline 7 & KBS & 220 & 2009 & 853 & Ghent U & 139 & France & 517 & Torra V & 71 \\
\hline 8 & IJUFKS & 188 & 2008 & 732 & $\begin{array}{l}\text { Southwest } \\
\text { Jiaotong U }\end{array}$ & 118 & Canada & 472 & Ma ZM & 66 \\
\hline 9 & JIFS & 176 & 2007 & 697 & Tsinghua U & 111 & Poland & 374 & Kacprzyk J & 66 \\
\hline 10 & SCO & 149 & 2006 & 546 & Xi'an Jiaotong U & 106 & Australia & 306 & $\mathrm{Xu} \mathrm{Y}$ & 65 \\
\hline 11 & ASC & 140 & 2005 & 450 & U Barcelona & 102 & Japan & 300 & Prade $\mathrm{H}$ & 65 \\
\hline 12 & EJOR & 100 & 2004 & 374 & City U Hong Kong & 97 & Germany & 264 & Dubois D & 64 \\
\hline 13 & IJCIS & 81 & 2003 & 391 & CSIC & 93 & India & 263 & Montero J & 62 \\
\hline 14 & IJGS & 79 & 2002 & 297 & $\begin{array}{l}\text { Northeastern U } \\
\text { China }\end{array}$ & 92 & Iran & 257 & Wei GW & 61 \\
\hline 15 & IEEETSMCB & 79 & 2001 & 289 & U Manchester & 85 & Belgium & 244 & Liu J & 61 \\
\hline 16 & ARIN & 63 & 2000 & 230 & $\begin{array}{l}\text { U Paul Sabatier } \\
\text { Toulouse III }\end{array}$ & 83 & Turkey & 240 & Pedrycz W & 55 \\
\hline 17 & CIE & 62 & 1999 & 196 & $\begin{array}{l}\text { Chinese Academy } \\
\text { Sciences }\end{array}$ & 83 & $\begin{array}{l}\text { South } \\
\text { Korea }\end{array}$ & 189 & Bustince $\mathrm{H}$ & 55 \\
\hline 18 & IEEETKDE & 61 & 1998 & 170 & Sichuan U & 81 & $\begin{array}{l}\text { The } \\
\text { Nether- } \\
\text { lands }\end{array}$ & 148 & Liu XW & 54 \\
\hline 19 & FIN & 60 & 1997 & 159 & $\begin{array}{l}\text { Shanghai Jiao } \\
\text { Tong U }\end{array}$ & 79 & $\begin{array}{l}\text { Czech } \\
\text { Republic }\end{array}$ & 134 & Chen XH & 54 \\
\hline 20 & FAIA & 58 & 1996 & 157 & $\begin{array}{l}\text { PLA U Science } \\
\text { and Technology }\end{array}$ & 79 & Brazil & 126 & Ruan D & 52 \\
\hline 21 & MPE & 55 & 1995 & 121 & Central South U & 79 & Greece & 112 & Zadrozny S & 49 \\
\hline 22 & EAAI & 51 & 1994 & 103 & Islamic Azad U & 78 & Malaysia & 101 & Wang J & 47 \\
\hline 23 & AMM & 51 & 1993 & 56 & U Bristol & 77 & Israel & 93 & Chiclana F & 47 \\
\hline 24 & AIN & 51 & 1992 & 55 & CNR & 71 & Singapore & 88 & Zhang WX & 45 \\
\hline
\end{tabular}


Table III. Continued

\begin{tabular}{|c|c|c|c|c|c|c|c|c|c|c|}
\hline $\mathrm{R}$ & Journals & TP & Years & $\mathrm{TP}$ & Universities & $\mathrm{TP}$ & Countries & $\mathrm{TP}$ & $\begin{array}{l}\text { Name of } \\
\text { authors }\end{array}$ & $\mathrm{TP}$ \\
\hline 25 & NEU & 46 & 1991 & 34 & U Alberta & 70 & Sweden & 72 & Vila MA & 44 \\
\hline 26 & PRE & 44 & 1990 & 17 & $\begin{array}{l}\text { Chinese U Hong } \\
\text { Kong }\end{array}$ & 70 & Mexico & 67 & Mesiar R & 44 \\
\hline 27 & JAM & 42 & 1989 & 7 & $\begin{array}{l}\text { Complutense U } \\
\text { Madrid }\end{array}$ & 66 & Tunisia & 60 & Chen HY & 44 \\
\hline 28 & IF & 40 & 1988 & 5 & $\begin{array}{c}\text { National Taiwan } \\
\text { U Science and } \\
\text { Technology }\end{array}$ & 65 & Switzerland & 56 & Wu WZ & 41 \\
\hline 29 & IEEETSMCA & 40 & 1987 & 1 & $\begin{array}{l}\text { Chongqing U Arts } \\
\text { and Sciences }\end{array}$ & 60 & $\begin{array}{l}\text { Saudi } \\
\text { Arabia }\end{array}$ & 54 & Qian YH & 41 \\
\hline 30 & NCA & 39 & - & - & $\begin{array}{l}\text { Harbin Institute of } \\
\text { Technology }\end{array}$ & 59 & Hungary & 54 & Zhou LG & 40 \\
\hline
\end{tabular}

Source: Own elaboration based on WoS. TP: Total publications; ISC: Information Sciences; IJIS: International Journal of Intelligent Systems; FSS: Fuzzy Sets and Systems; ESA: Expert Systems with Applications; IEEETFS: IEEE Transactions Fuzzy Systems; IJAR: International Journal of Approximate Reasoning; KBS: Knowledge-Based Systems; IJUFKBS: International Journal of Uncertainty Fuzziness and Knowledge Based Systems; JIFS: Journal of Intelligent \& Fuzzy Systems; SCO: Soft Computing; ASC: Applied Soft Computing; EJOR: European Journal of Operational Research; IJCIS: International Journal of Computational Intelligence Systems; IJGS: International Journal of General Systems; IEEETSMCB: IEEE Transactions Of Systems Man and Cybernetics, Part B Cybernetics; ARIN: Artificial Intelligence; CIE: Computers Industrial Engineering; IEEETKDE: IEEE Transactions of Knowledge and Data Engineering; FIN: Fundamenta Informaticae; FAIA: Frontier in Artificial Intelligence and Applications; MPE: Mathematical Problems in Engineering; EAAI: Engineering Applications of Artificial Intelligence; AMM: Applied Mathematical Modelling; AIN: Applied Intelligence; NEU: Neurocomputing; PRE: Pattern Recognition; JAM: Journal of Applied Mathematics; IF: Information Fusion; IEEETSMCA: IEEE Transactions of Systems Man and Cybernetics, Part A Systems and Humans; NCA: Neural Computing \& Applications; CNRS: National Research Council - France; CSIC: National Research Council - Spain; CNR: National Research Council - Italy.

contributions that have led the journal. First, let us initially analyze the work of the most productive and influential authors. Table IV presents the 50 most influential authors in the journal, with a minimum publication level of five papers. Thus, the ranking is according to citations but only considering those authors who have published at least five documents in IJIS.

Zeshui $\mathrm{Xu}$ is the author that has received the highest influence based on his papers published in the journal. Yager appears in the second position, although he is the most productive author in the journal. Note that $\mathrm{Xu}$ and Herrera have published six papers among the 50 most cited in the journal. Additionally, these three authors are very highly cited, particularly Herrera and Yager, who have more than 15,000 citations in WoS, which implies that they are very highly ranked in this field worldwide. Note that other very highly cited researchers have also influenced the journal significantly including Henri Prade, Didier Dubois, Enrique HerreraViedma, Witold Pedrycz, and James C. Bezdek.

Next, let us examine the productivity of the leading authors over time. Table $\mathrm{V}$ presents the evolution of the publications of the most productive authors in the journal. Note that the publications before 1996 are summarized in two groups of 5 years. 
MERIGÓ ET AL.

Table IV. Most-productive and influential authors in IJIS.

\begin{tabular}{|c|c|c|c|c|c|c|c|c|c|c|c|}
\hline \multirow[b]{2}{*}{$\mathrm{R}$} & \multirow[b]{2}{*}{ Name of authors } & \multirow[b]{2}{*}{ Country } & \multicolumn{5}{|c|}{ IJIS } & \multicolumn{4}{|c|}{ Total } \\
\hline & & & $\mathrm{TC}$ & $\mathrm{TP}$ & $\mathrm{TC} / \mathrm{TP}$ & $\mathrm{H}$ & $\mathrm{T} 50$ & $\mathrm{TC}$ & $\mathrm{TP}$ & $\mathrm{TC} / \mathrm{TP}$ & $\mathrm{H}$ \\
\hline 1 & $\mathrm{Xu}$ ZS & $\mathrm{CHN}$ & 1466 & 25 & 58.64 & 14 & 6 & 8136 & 226 & 36.00 & 48 \\
\hline 2 & Yager RR & USA & 1168 & 37 & 31.57 & 18 & 3 & 16316 & 549 & 29.72 & 58 \\
\hline 3 & Herrera F & ESP & 830 & 20 & 41.5 & 14 & 6 & 17965 & 399 & 45.03 & 69 \\
\hline 4 & Torra V & SWE & 775 & 24 & 32.29 & 8 & 3 & 1941 & 183 & 10.61 & 21 \\
\hline 5 & Vila MA & ESP & 550 & 17 & 32.35 & 9 & 2 & 2044 & 159 & 12.86 & 21 \\
\hline 6 & Prade H & FRA & 509 & 26 & 19.58 & 12 & 2 & 12935 & 537 & 24.09 & 57 \\
\hline 7 & Dubois D & FRA & 485 & 17 & 28.53 & 12 & 2 & 11110 & 355 & 31.30 & 56 \\
\hline 8 & Herrera-Viedma E & ESP & 462 & 11 & 42 & 7 & 5 & 9697 & 275 & 35.26 & 51 \\
\hline 9 & Verdegay JL & ESP & 428 & 9 & 47.56 & 6 & 2 & 3630 & 109 & 33.30 & 26 \\
\hline 10 & Delgado M & ESP & 421 & 18 & 23.39 & 6 & 2 & 1537 & 151 & 10.18 & 16 \\
\hline 11 & Chiclana F & UK & 418 & 8 & 52.25 & 7 & 4 & 3961 & 89 & 44.51 & 27 \\
\hline 12 & Ruan D & BEL & 363 & 19 & 19.11 & 11 & 1 & 1249 & 116 & 10.77 & 21 \\
\hline 13 & Lozano M & ESP & 267 & 6 & 44.5 & 5 & 1 & 2544 & 56 & 45.43 & 24 \\
\hline 14 & Pedrycz W & $\mathrm{CAN}$ & 224 & 18 & 12.44 & 8 & - & 10535 & 882 & 11.94 & 47 \\
\hline 15 & Kacprzyk J & POL & 191 & 8 & 23.88 & 6 & 1 & 3388 & 245 & 13.83 & 26 \\
\hline 16 & Smets P & BEL & 181 & 5 & 36.2 & 5 & 2 & 2871 & 68 & 42.22 & 25 \\
\hline 17 & Kahraman C & TUR & 179 & 8 & 22.38 & 6 & 1 & 3554 & 230 & 15.45 & 33 \\
\hline 18 & Merigo JM & $\mathrm{CHL}$ & 177 & 5 & 35.4 & 3 & 2 & 1771 & 135 & 13.12 & 23 \\
\hline 19 & Pasi G & ITA & 163 & 10 & 16.3 & 6 & 2 & 1082 & 90 & 12.02 & 13 \\
\hline 20 & Mitchell HB & ISR & 158 & 10 & 15.8 & 7 & - & 589 & 33 & 17.85 & 12 \\
\hline 21 & Moral S & ESP & 146 & 12 & 12.17 & 6 & - & 1042 & 115 & 9.06 & 19 \\
\hline 22 & Bordogna $\mathrm{G}$ & ITA & 143 & 6 & 23.83 & 4 & - & 1139 & 88 & 12.94 & 16 \\
\hline 23 & Bezdek JC & AUS & 131 & 6 & 21.83 & 4 & 1 & 9286 & 209 & 44.43 & 45 \\
\hline 24 & Ma ZM & $\mathrm{CHN}$ & 120 & 8 & 15 & 6 & - & 612 & 89 & 6.88 & 14 \\
\hline 25 & Cubero JC & ESP & 110 & 6 & 18.33 & 4 & - & 379 & 45 & 8.42 & 11 \\
\hline 26 & Grabisch M & FRA & 101 & 8 & 12.63 & 6 & - & 2670 & 140 & 19.07 & 27 \\
\hline 27 & Schaefer PA & ISR & 99 & 6 & 16.5 & 5 & - & 120 & 10 & 12.00 & 6 \\
\hline 28 & Pons O & ESP & 98 & 9 & 10.89 & 6 & - & 351 & 39 & 9.00 & 10 \\
\hline 29 & Martinez L & ESP & 98 & 6 & 16.33 & 3 & - & 3904 & 118 & 33.08 & 26 \\
\hline 30 & Yang MS & TWN & 93 & 8 & 11.63 & 5 & - & 2261 & 112 & 20.19 & 26 \\
\hline 31 & Kreinovich V & USA & 87 & 22 & 3.95 & 5 & - & 1213 & 377 & 3.22 & 18 \\
\hline 32 & Kerre EE & BEL & 87 & 9 & 9.67 & 5 & - & 3428 & 203 & 16.89 & 29 \\
\hline 33 & Trillas E & ESP & 79 & 7 & 11.29 & 5 & - & 901 & 115 & 7.83 & 15 \\
\hline 34 & Ford KM & USA & 78 & 6 & 13 & 4 & - & 160 & 26 & 6.15 & 8 \\
\hline 35 & $\mathrm{Li} \mathrm{TR}$ & $\mathrm{CHN}$ & 78 & 5 & 15.6 & 3 & - & 808 & 142 & 5.69 & 17 \\
\hline 36 & Nguyen HT & USA & 77 & 11 & 7 & 5 & - & 610 & 95 & 6.42 & 12 \\
\hline 37 & Chen GQ & $\mathrm{CHN}$ & 73 & 7 & 10.43 & 3 & - & 745 & 102 & 7.30 & 14 \\
\hline 38 & Liu XW & $\mathrm{CHN}$ & 68 & 5 & 13.6 & 4 & - & 908 & 77 & 11.79 & 18 \\
\hline 39 & Medina JM & ESP & 67 & 6 & 11.17 & 4 & - & 339 & 36 & 9.42 & 9 \\
\hline 40 & Kandel A & USA & 64 & 17 & 3.76 & 5 & - & 3634 & 333 & 10.91 & 30 \\
\hline 41 & Sun $\mathrm{ZH}$ & $\mathrm{CHN}$ & 62 & 6 & 10.33 & 4 & - & 134 & 22 & 6.09 & 14 \\
\hline 42 & Finnie G & AUS & 61 & 5 & 12.2 & 4 & - & 294 & 28 & 10.50 & 8 \\
\hline 43 & Marin N & ESP & 59 & 5 & 11.8 & 5 & - & 407 & 51 & 7.98 & 11 \\
\hline 44 & Srivastava RP & USA & 58 & 6 & 9.67 & 5 & - & 207 & 28 & 7.39 & 9 \\
\hline 45 & Lee $\mathrm{J}$ & TWN & 57 & 13 & 4.38 & 5 & - & 325 & 40 & 8.13 & 10 \\
\hline 46 & Keller JM & USA & 54 & 7 & 7.71 & 5 & - & 2979 & 228 & 13.56 & 26 \\
\hline 47 & Baldwin JF & UK & 54 & 7 & 7.71 & 4 & - & 722 & 79 & 9.14 & 14 \\
\hline
\end{tabular}

(Continued) 
Table IV. Continued

\begin{tabular}{|c|c|c|c|c|c|c|c|c|c|c|c|}
\hline \multirow[b]{2}{*}{$\mathrm{R}$} & \multirow[b]{2}{*}{ Name of authors } & \multirow[b]{2}{*}{ Country } & \multicolumn{5}{|c|}{ IJIS } & \multicolumn{4}{|c|}{ Total } \\
\hline & & & $\mathrm{TC}$ & $\mathrm{TP}$ & $\mathrm{TC} / \mathrm{TP}$ & $\mathrm{H}$ & $\mathrm{T} 50$ & $\mathrm{TC}$ & $\mathrm{TP}$ & $\mathrm{TC} / \mathrm{TP}$ & $\mathrm{H}$ \\
\hline 48 & Vanthienen $\mathrm{J}$ & BEL & 54 & 5 & 10.8 & 3 & - & 1467 & 123 & 11.93 & 17 \\
\hline 49 & Loia V & ITA & 51 & 7 & 7.29 & 3 & - & 881 & 169 & 5.21 & 16 \\
\hline 50 & Wang ZY & USA & 51 & 5 & 10.2 & 3 & - & 218 & 30 & 7.27 & 8 \\
\hline
\end{tabular}

Source: Own elaboration based on WoS. H: $h$-index; TP: total publications; TC: total citations; T50 = number of papers in the list of Table II; ELTA S*: Israel Aerospace Industries Ltd.

As seen, with very few exceptions, it is uncommon for an author to publish more than one paper in the same year. In particular, note the case of Zeshui Xu, who has published at least two papers every year since 2010. Moreover, the table also shows the citation thresholds that these papers have reached to date.

Many institutions have a great deal of influence in IJIS. Usually, the leading institutions are those at which the leading authors work. However, occasionally, some institutions include a wide range of leading authors, making them more influential. Table VI shows the 50 most productive institutions in IJIS. Note that the table also considers citations, citations per paper, and the $h$-index.

The University of Granada obtains the most remarkable results, with a publication volume much higher than other institutions. Note that this difference is also seen in Table IV, which shows that five of the Top 10 authors work at the University of Granada. Many others are also seen in the Top 50 or working at another institution but with close connections. There is a huge imbalance in the list, which includes many English-speaking and European institutions. Note that this imbalance is quite common in science and technology but not in social sciences, in which English-speaking countries tend to have a stronger dominance in the rankings. ${ }^{4,6}$

When developing the institutional analysis, it is also interesting to see the evolution over time. Table VII presents the temporal evolution of the most productive institutions.

As seen, for most of the time, the University of Granada has been the most productive university in the journal with the exception of the first 10 years, when the CNRS (France) and Iona College (USA) had more-significant results.

Another interesting issue to consider is to classify the authors and institutions by countries. Thus, we can see the regions in which IJIS is more influential. To perform this classification, Table VIII presents the 30 most productive countries in the journals. The countries are ranked by total productivity, although other indicators are considered including citations and productivity and influence per person.

The USA, Spain, and China are the leading countries in IJIS. Note the case of Spain, because it is a much smaller country compared with the other two. These positions can be observed by examining the per capita results, in which Spain obtains a very remarkable rate. Note that Belgium also obtains results similar to Spain. However, because it is four times smaller, it does not occupy a significant 


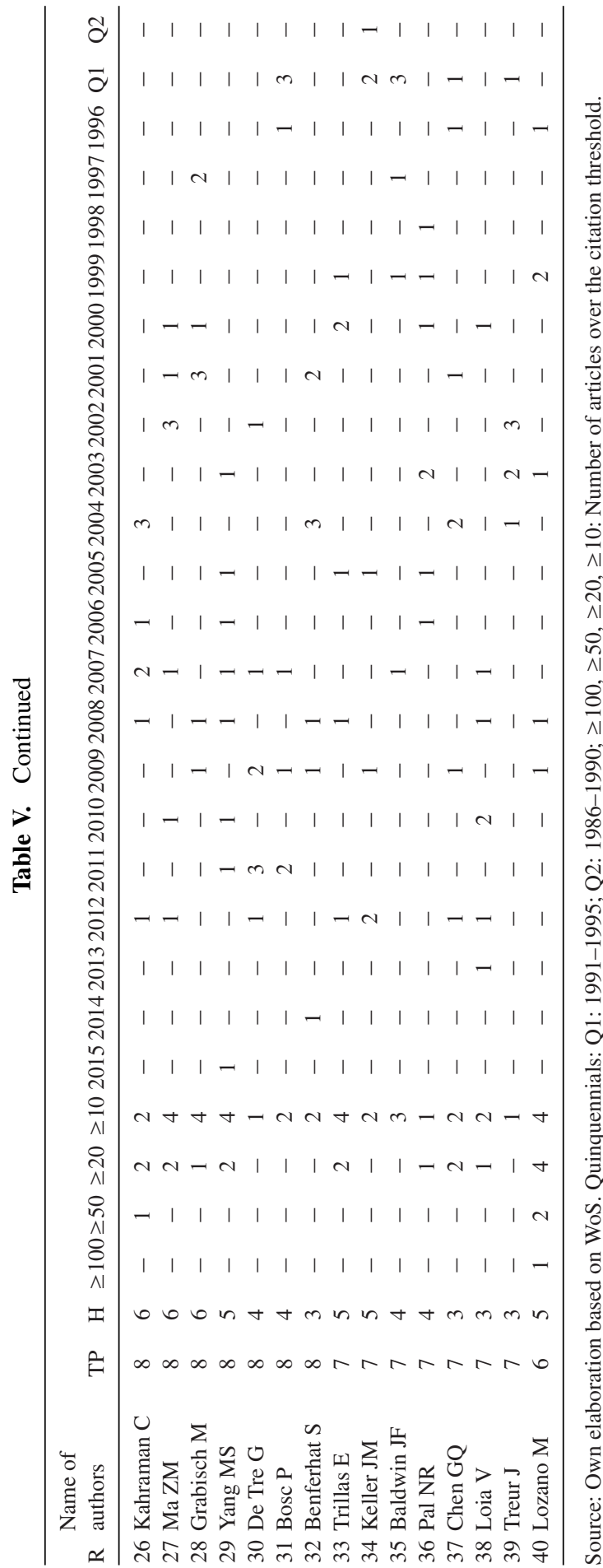


MERIGÓ ET AL.

Table VI. 50 most productive institutions in IJIS.

\begin{tabular}{|c|c|c|c|c|c|c|c|c|c|}
\hline $\mathrm{R}$ & Universities & Country & $\mathrm{TP}$ & $\mathrm{H}$ & $\mathrm{TC}$ & $\mathrm{TC} / \mathrm{TP}$ & ARWU & QS & $\begin{array}{c}\text { TOP } \\
50\end{array}$ \\
\hline 1 & U Granada & ESP & 91 & 22 & 1814 & 19.93 & $301-400$ & $461-470$ & 9 \\
\hline 2 & CNRS & FRA & 44 & 14 & 631 & 14.34 & - & - & 2 \\
\hline 3 & Iona College & USA & 40 & 16 & 1088 & 27.20 & - & - & 3 \\
\hline 6 & CSIC & ESP & 31 & 10 & 570 & 18.39 & - & - & 1 \\
\hline 4 & $\begin{array}{l}\text { Polish Academy } \\
\text { of Sciences }\end{array}$ & POL & 31 & 12 & 422 & 13.61 & - & - & 1 \\
\hline 5 & $\begin{array}{l}\text { U Paul Sabatier } \\
\text { Toulouse III }\end{array}$ & FRA & 29 & 13 & 420 & 14.48 & $201-300$ & 397 & 1 \\
\hline 7 & U Texas El Paso & USA & 24 & 6 & 95 & 3.96 & - & - & - \\
\hline 8 & $\begin{array}{l}\text { Universidad } \\
\text { Politécnica de } \\
\text { Madrid }\end{array}$ & ESP & 22 & 8 & 209 & 9.50 & - & $461-470$ & 1 \\
\hline 9 & Ghent U & BEL & 22 & 9 & 201 & 9.14 & 71 & 124 & - \\
\hline 10 & Southeast U & $\mathrm{CHN}$ & 21 & 13 & 1303 & 62.05 & $301-400$ & $461-470$ & 5 \\
\hline 11 & $\begin{array}{l}\text { Chinese U Hong } \\
\text { Kong }\end{array}$ & $\mathrm{CHN}$ & 18 & 7 & 155 & 8.61 & - & 51 & - \\
\hline 12 & $\begin{array}{l}\text { New Mexico State } \\
\text { U }\end{array}$ & USA & 17 & 6 & 191 & 11.24 & - & - & 1 \\
\hline 13 & U South Florida & USA & 17 & 5 & 60 & 3.53 & $201-300$ & $501-550$ & - \\
\hline 14 & U Alberta & CAN & 16 & 7 & 191 & 11.94 & $101-150$ & 96 & - \\
\hline 15 & U Bristol & UK & 16 & 6 & 128 & 8.00 & 66 & 37 & - \\
\hline 16 & $\begin{array}{l}\text { National Central } \\
\quad \mathrm{U}\end{array}$ & TWN & 16 & 5 & 80 & 5.00 & - & 397 & - \\
\hline 17 & U Jaen & ESP & 15 & 8 & 320 & 21.33 & - & - & 2 \\
\hline 18 & CEN SCK & BEL & 15 & 9 & 266 & 17.73 & - & - & 1 \\
\hline 19 & CNR & ITA & 15 & 7 & 221 & 14.73 & - & - & 2 \\
\hline 20 & U Salerno & ITA & 15 & 5 & 107 & 7.13 & - & - & - \\
\hline 21 & $\begin{array}{l}\text { Pierre Marie Curie } \\
\text { U Paris VI }\end{array}$ & FRA & 14 & 7 & 139 & 9.93 & 36 & 137 & - \\
\hline 24 & U North Carolina & USA & 14 & 5 & 74 & 5.29 & 39 & 79 & - \\
\hline 22 & $\begin{array}{c}\text { U California } \\
\text { Berkeley }\end{array}$ & USA & 13 & 6 & 151 & 11.62 & 4 & 26 & - \\
\hline 23 & U Ulster & UK & 13 & 6 & 147 & 11.31 & - & $551-600$ & - \\
\hline 25 & $\begin{array}{l}\text { Universidad } \\
\text { Politécnica de } \\
\text { Catalonia }\end{array}$ & ESP & 13 & 4 & 61 & 4.69 & $401-500$ & 299 & - \\
\hline 26 & Tsinghua U & $\mathrm{CHN}$ & 12 & 5 & 145 & 12.08 & $101-150$ & 25 & - \\
\hline 27 & U Kansas & USA & 12 & 6 & 125 & 10.42 & $79-102$ & 393 & - \\
\hline 28 & U Almeria & ESP & 12 & 3 & 61 & 5.08 & - & - & - \\
\hline 29 & $\begin{array}{l}\text { Universitat } \\
\text { Autonoma } \\
\text { Barcelona }\end{array}$ & ESP & 12 & 4 & 51 & 4.25 & $201-300$ & 190 & - \\
\hline 30 & U West Florida & USA & 11 & 6 & 188 & 17.09 & - & - & 1 \\
\hline 31 & $\begin{array}{l}\text { Bulgarian } \\
\text { Academy of } \\
\text { Sciences }\end{array}$ & BUL & 11 & 3 & 131 & 11.91 & - & - & 1 \\
\hline 32 & $\begin{array}{l}\text { U Missouri } \\
\text { Columbia }\end{array}$ & USA & 11 & 6 & 63 & 5.73 & $103-125$ & $501-550$ & - \\
\hline 33 & $\begin{array}{l}\text { U Libre de } \\
\text { Bruxelles }\end{array}$ & BEL & 10 & 6 & 204 & 20.40 & $101-150$ & 207 & 2 \\
\hline
\end{tabular}

(Continued) 
Table VI. Continued

\begin{tabular}{|c|c|c|c|c|c|c|c|c|c|}
\hline $\mathrm{R}$ & Universities & Country & TP & $\mathrm{H}$ & $\mathrm{TC}$ & TC/TP & ARWU & QS & $\begin{array}{c}\text { TOP } \\
50\end{array}$ \\
\hline 34 & $\begin{array}{l}\text { U LE Mirail } \\
\text { Toulouse II }\end{array}$ & FRA & 10 & 5 & 198 & 19.80 & - & $701+$ & 1 \\
\hline 35 & $\begin{array}{l}\text { U Naples Federico } \\
\text { II }\end{array}$ & ITA & 10 & 6 & 92 & 9.20 & - & $441-450$ & - \\
\hline 36 & $\begin{array}{l}\text { U Southern } \\
\text { California }\end{array}$ & USA & 10 & 3 & 75 & 7.50 & 49 & 130 & - \\
\hline 37 & U Manitoba & CAN & 10 & 5 & 73 & 7.30 & $301-400$ & $551-600$ & - \\
\hline 38 & De Montfort U & UK & 9 & 7 & 423 & 47.00 & - & - & 4 \\
\hline 39 & $\begin{array}{l}\text { PLA U Science } \\
\text { and Technology }\end{array}$ & $\mathrm{CHN}$ & 9 & 5 & 321 & 35.67 & - & - & 2 \\
\hline 40 & $\begin{array}{l}\text { Institut National } \\
\text { Polytechnique } \\
\text { de Toulouse }\end{array}$ & FRA & 9 & 4 & 184 & 20.44 & - & - & 1 \\
\hline 41 & $\begin{array}{l}\text { Southwest } \\
\text { Jiaotong U }\end{array}$ & $\mathrm{CHN}$ & 9 & 7 & 148 & 16.44 & - & - & - \\
\hline 42 & U Balearic Islands & ESP & 9 & 5 & 132 & 14.67 & - & - & 1 \\
\hline 43 & $\begin{array}{c}\text { Czech Academy } \\
\text { of Sciences }\end{array}$ & CZE & 9 & 6 & 79 & 8.78 & - & - & - \\
\hline 44 & U Murcia & ESP & 9 & 4 & 50 & 5.56 & - & $701+$ & - \\
\hline 45 & U Tsukuba & JPN & 9 & 3 & 49 & 5.44 & $201-300$ & 219 & - \\
\hline 46 & $\begin{array}{l}\text { U Maryland } \\
\text { College Park }\end{array}$ & USA & 9 & 4 & 45 & 5.00 & 43 & 126 & - \\
\hline 47 & Ben Gurion U & ISR & 9 & 3 & 31 & 3.44 & $401-500$ & 259 & - \\
\hline 48 & Nat U Singapore & SGP & 9 & 3 & 29 & 3.22 & $101-150$ & 12 & - \\
\hline 49 & U Toulouse I & FRA & 8 & 4 & 183 & 22.88 & - & - & 1 \\
\hline 50 & Istanbul Teknik U & TUR & 8 & 6 & 179 & 22.38 & - & $551-600$ & 1 \\
\hline
\end{tabular}

Source: Own elaboration based on WoS. ARWU: Academic ranking of world universities (only the top 500); QS: world university rankings (only the top 800); organization abbreviations are available in Table I except for; CEN SCK: Belgium Centre of Nuclear Research.

position at the top of the list. Observe that some developing countries also appear in the list including India, Turkey, Brazil, Iran, Mexico, and Thailand. Currently, they do not have significant positions, although expectations for the future are that they will continue growing significantly, reaching results similar to those that China has already obtained.

Next, let us examine the productivity of countries over time. Table IX presents the temporal evolution of the publications of the 30 countries that appeared in Table VIII. During the first years of the journal, the USA was the most productive country. However, Spain and China are now publishing more. Spain has had a more stable evolution over time, whereas China has been increasing its productivity significantly in recent years; currently, it is the most productive country in the journal.

To obtain a more general perspective of the country analysis, Table $\mathrm{X}$ analyzes the countries grouped by continent. Note that for some huge continents, the table also divides between smaller supraregions to obtain a deeper perspective of each region. 


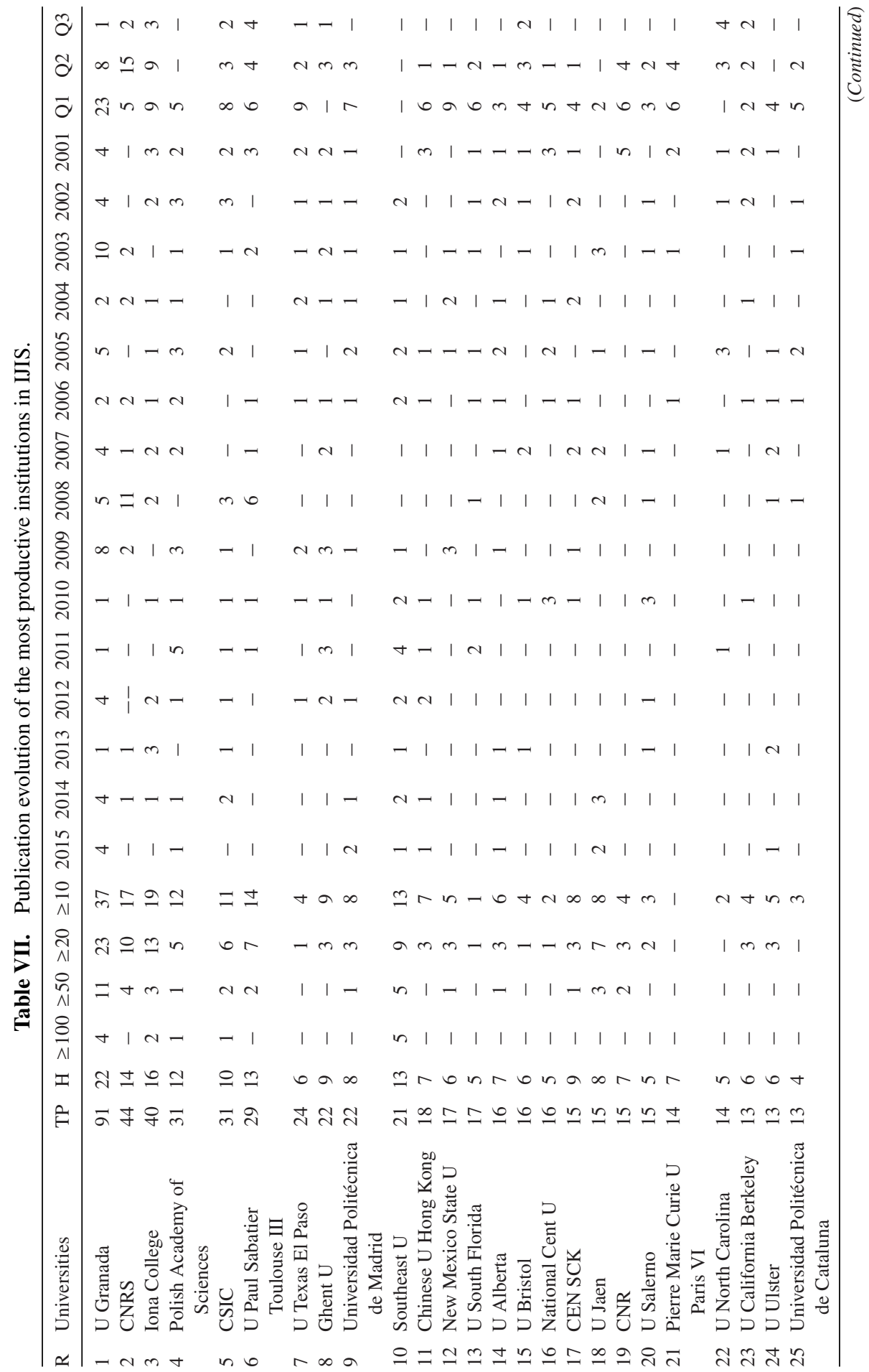




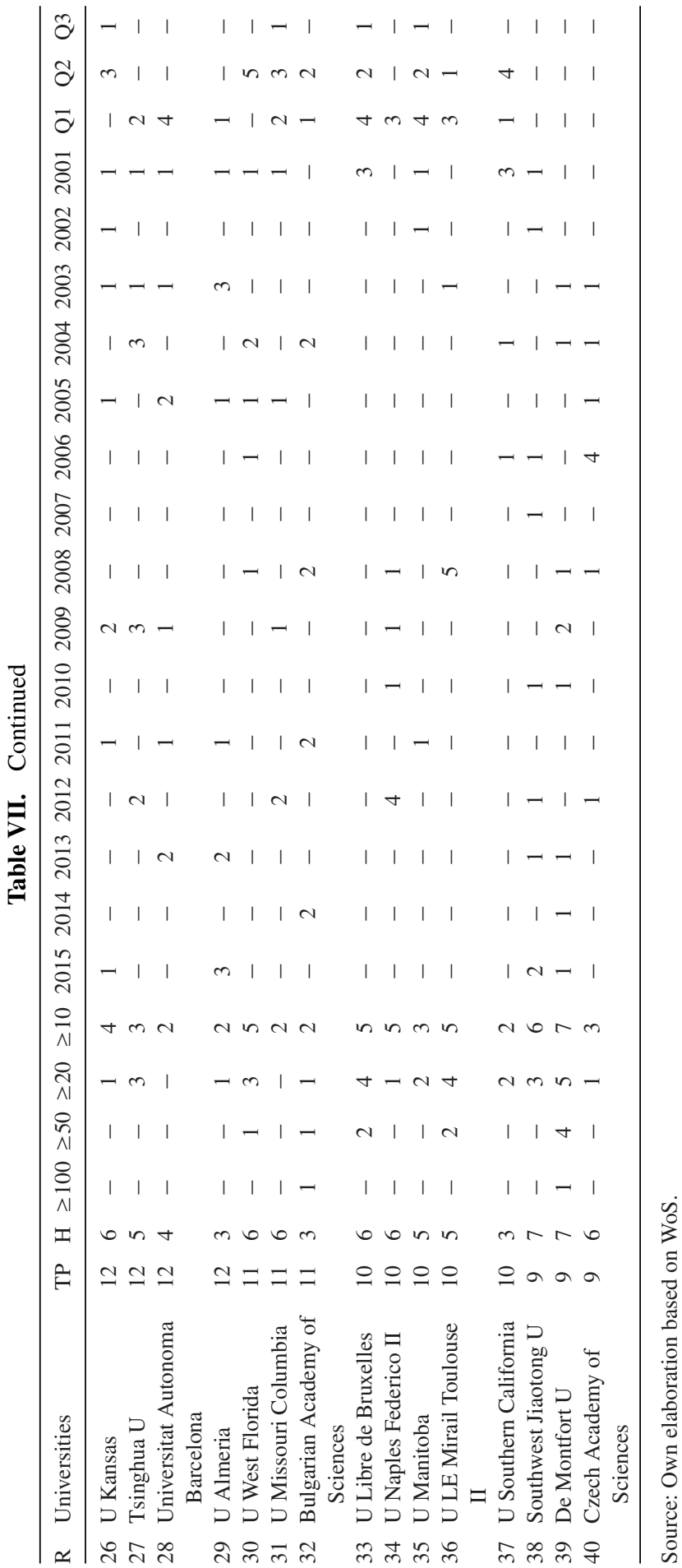

International Journal of Intelligent Systems 
Table VIII. Most productive and influential countries in IJIS.

\begin{tabular}{|c|c|c|c|c|c|c|c|c|c|}
\hline $\mathrm{R}$ & Countries & $\mathrm{TP}$ & $\mathrm{H}$ & $\mathrm{TC}$ & TC/TP & Population & TP/Pop & TC/Pop & TOP 50 \\
\hline 1 & USA & 422 & 29 & 3589 & 8.50 & $318,857,056$ & 1.32 & 11.26 & 8 \\
\hline 2 & Spain & 238 & 33 & 3855 & 16.20 & $46,404,602$ & 5.13 & 83.07 & 18 \\
\hline 3 & People's Republic of China & 178 & 26 & 2755 & 15.48 & $1,364,270,000$ & 0.13 & 2.02 & 11 \\
\hline 4 & Italy & 110 & 17 & 1066 & 9.69 & $61,336,387$ & 1.79 & 17.38 & 4 \\
\hline 5 & France & 106 & 18 & 1110 & 10.47 & $66,206,930$ & 1.60 & 16.77 & 3 \\
\hline 6 & UK & 98 & 16 & 1105 & 11.28 & $64,510,376$ & 1.52 & 17.13 & 5 \\
\hline 7 & Canada & 70 & 16 & 831 & 11.87 & $35,540,419$ & 1.97 & 23.38 & 1 \\
\hline 8 & Taiwan & 61 & 11 & 428 & 7.02 & $23,434,000$ & 2.60 & 18.26 & 1 \\
\hline 9 & Japan & 60 & 9 & 376 & 6.27 & $127,131,800$ & 0.47 & 2.96 & 1 \\
\hline 10 & Belgium & 59 & 16 & 805 & 13.64 & $11,225,207$ & 5.26 & 71.71 & 3 \\
\hline 11 & Poland & 51 & 16 & 1040 & 20.39 & $37,995,529$ & 1.34 & 27.37 & 5 \\
\hline 12 & Germany & 44 & 11 & 309 & 7.02 & $80,889,505$ & 0.54 & 3.82 & - \\
\hline 13 & Australia & 41 & 12 & 535 & 13.05 & $23,490,736$ & 1.75 & 22.77 & 2 \\
\hline 14 & Israel & 30 & 8 & 214 & 7.13 & $8,215,300$ & 3.65 & 26.05 & - \\
\hline 15 & South Korea & 28 & 9 & 239 & 8.54 & $50,423,955$ & 0.56 & 4.74 & - \\
\hline 16 & India & 28 & 6 & 119 & 4.25 & $1,295,291,543$ & 0.02 & 0.09 & - \\
\hline 17 & Greece & 28 & 6 & 83 & 2.96 & $10,957,740$ & 2.56 & 7.57 & - \\
\hline 18 & Turkey & 26 & 9 & 307 & 11.81 & $75,932,348$ & 0.34 & 4.04 & 1 \\
\hline 19 & The Netherlands & 25 & 7 & 128 & 5.12 & $16,854,183$ & 1.48 & 7.59 & - \\
\hline 20 & Sweden & 17 & 6 & 130 & 7.65 & $9,689,555$ & 1.75 & 13.42 & - \\
\hline 21 & Singapore & 17 & 5 & 106 & 6.24 & $5,469,700$ & 3.11 & 19.38 & - \\
\hline 22 & Czech Republic & 17 & 7 & 102 & 6.00 & $10,510,566$ & 1.62 & 9.70 & - \\
\hline 23 & Brazil & 13 & 4 & 78 & 6.00 & $206,077,898$ & 0.06 & 0.38 & - \\
\hline 24 & Iran & 13 & 4 & 51 & 3.92 & $78,143,644$ & 0.17 & 0.65 & - \\
\hline 25 & Bulgaria & 12 & 3 & 132 & 11.00 & $7,226,291$ & 1.66 & 18.27 & 1 \\
\hline 26 & Mexico & 10 & 5 & 75 & 7.50 & $125,385,833$ & 0.08 & 0.60 & - \\
\hline 27 & Denmark & 10 & 5 & 72 & 7.20 & $5,639,565$ & 1.77 & 12.77 & - \\
\hline 28 & Switzerland & 10 & 6 & 66 & 6.60 & $8,190,229$ & 1.22 & 8.06 & - \\
\hline 29 & Thailand & 10 & 3 & 21 & 2.10 & $67,725,979$ & 0.15 & 0.31 & - \\
\hline 30 & Hungary & 8 & 2 & 41 & 5.13 & $9,861,673$ & 0.81 & 4.16 & - \\
\hline
\end{tabular}

Source: Own elaboration based on WoS. Pop: Population; TP/Pop; (Tp/Pop)*1,000,000; TC/Pop; (TC/Pop)*1,000,000.

Western Europe is the most productive region in the journal followed, by North America, and Eastern Asia. Developing regions do not publish so much, although they also have a significant number of publications compared with other fields in which their publication rate is much lower. This result is particularly interesting when examining the temporal evolution, which shows the growth of developing nations. However, note that this growth is seen particularly in Eastern Asia (and is led by China) but not so much in the remaining regions, in which the results remain quite stable. Table XI shows the results.

As seen, Eastern Asia has grown significantly in the journal and now is the most productive region, although very close to Western Europe. North America has lost much of its presence in the journal, with a significant decrease over time. Note that the main reason is that IJIS has continued publishing a similar number of papers in recent years, even reducing the number a bit. Therefore, the significant increase of submissions to the journal from other countries has resulted in the USA publishing much less compared to other countries than it did previously. 


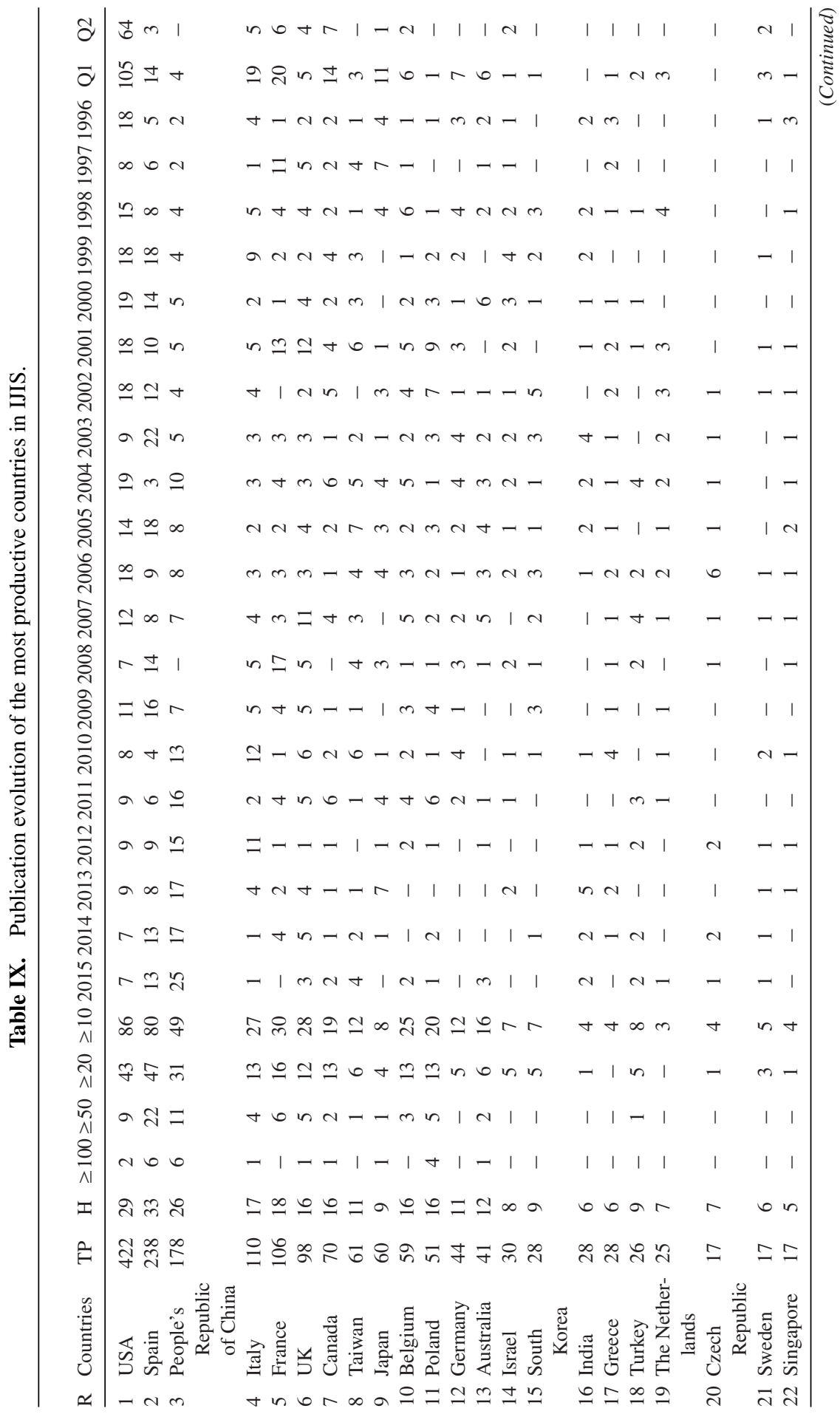

International Journal of Intelligent Systems 
MERIGÓ ET AL.

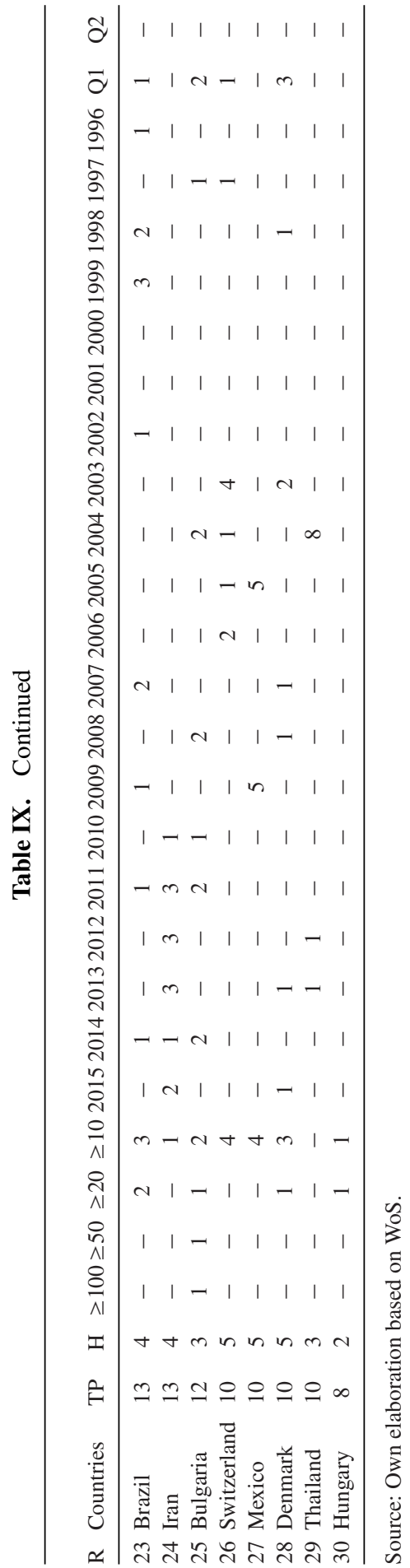

International Journal of Intelligent Systems 
Table X. Publications in IJIS classified by supraregions.

\begin{tabular}{|c|c|c|c|c|c|c|c|c|c|}
\hline $\mathrm{R}$ & Supraregions & $\mathrm{TP}$ & $\mathrm{H}$ & $\mathrm{TC}$ & TC/TP & Population & TP/Pop & TC/Pop & TOP 50 \\
\hline 1 & Europe & 771 & 44 & 8915 & 11.56 & $743,122,000$ & 1.038 & 11.997 & 33 \\
\hline- & Western, and North Europe & 688 & 41 & 7785 & 11.32 & $450,612,000$ & 1.527 & 17.277 & 28 \\
\hline- & Eastern Europe & 103 & 18 & 1373 & 13.33 & $292,500,000$ & 0.352 & 4.694 & 6 \\
\hline 2 & North America & 483 & 29 & 4253 & 8.81 & $354,462,656$ & 1.363 & 11.998 & 9 \\
\hline 3 & Latin America & 36 & 7 & 172 & 4.78 & $630,088,000$ & 0.057 & 0.273 & - \\
\hline 4 & Asia* & 461 & 32 & 4589 & 9.95 & $4,384,844,000$ & 0.105 & 1.047 & 14 \\
\hline- & Eastern Asia & 310 & 29 & 3879 & 12.51 & $2,264,079,901$ & 0.137 & 1.713 & 13 \\
\hline- & Southeast Asia & 31 & 7 & 176 & 5.68 & $633,031,000$ & 0.049 & 0.278 & - \\
\hline- & Western Asia & 87 & 14 & 628 & 7.22 & $254,466,000$ & 0.342 & 2.468 & 1 \\
\hline- & South Asia & 33 & 6 & 144 & 4.36 & $1,793,615,000$ & 0.018 & 0.080 & - \\
\hline 5 & Oceania & 47 & 12 & 549 & 11.68 & $39,359,000$ & 1.194 & 13.949 & 2 \\
\hline 6 & Africa & 6 & 3 & 23 & 3.83 & $1,166,239,000$ & 0.005 & 0.020 & - \\
\hline
\end{tabular}

Source: Own elaboration based on WoS: *Included Central Asia population.

\section{MAPPING IJIS THROUGH THE VOS VIEWER SOFTWARE}

To obtain a more complete picture of the leading trends occurring in IJIS, this section develops a graphical analysis of the leading issues in the journal. We use the VOS viewer software, ${ }^{20}$ which visualizes the bibliographic material through bibliographic coupling ${ }^{21}$ and co-citation analysis. ${ }^{22}$ Bibliographic coupling occurs when two documents published in IJIS cite the same third document. Note that the two connected documents appear in the figure but not the third one unless it also has a significant degree of bibliographic coupling through other documents. Co-citation occurs when two documents receive a citation by a third document that has been published in IJIS. Note that the figure visualizes the two documents that receive the citation but not the third document that cites the other two. Therefore, bibliographic coupling represents the highest productivity in the journal and represents how this research is connected. Moreover, co-citation shows the most-cited research in the journal and its connections. ${ }^{31}$

To perform the graphical analysis, let us focus initially on bibliographic coupling. Figure 2 presents the bibliographic coupling of the most productive authors in IJIS.

As seen, Yager is at the core of the graph, with the most significant connections. Herrera, Xu, Dubois, Prade, and Kandel also have significant positions.

Next, let us examine the bibliographic coupling of institutions in IJIS. Figure 3 shows the results. Note that the institutions have a strong connection with the authors because they represent the affiliation of the authors that publish in the journal.

The University of Granada is at the core of the graph. As was explained in Table VI, many leading authors in IJIS work at this institution. The Polish Academy of Sciences, the National Research Council of Spain (CSIC), Iona College, Southeast University (China), and the Chinese University of Hong Kong also have a significant position in the graph. 


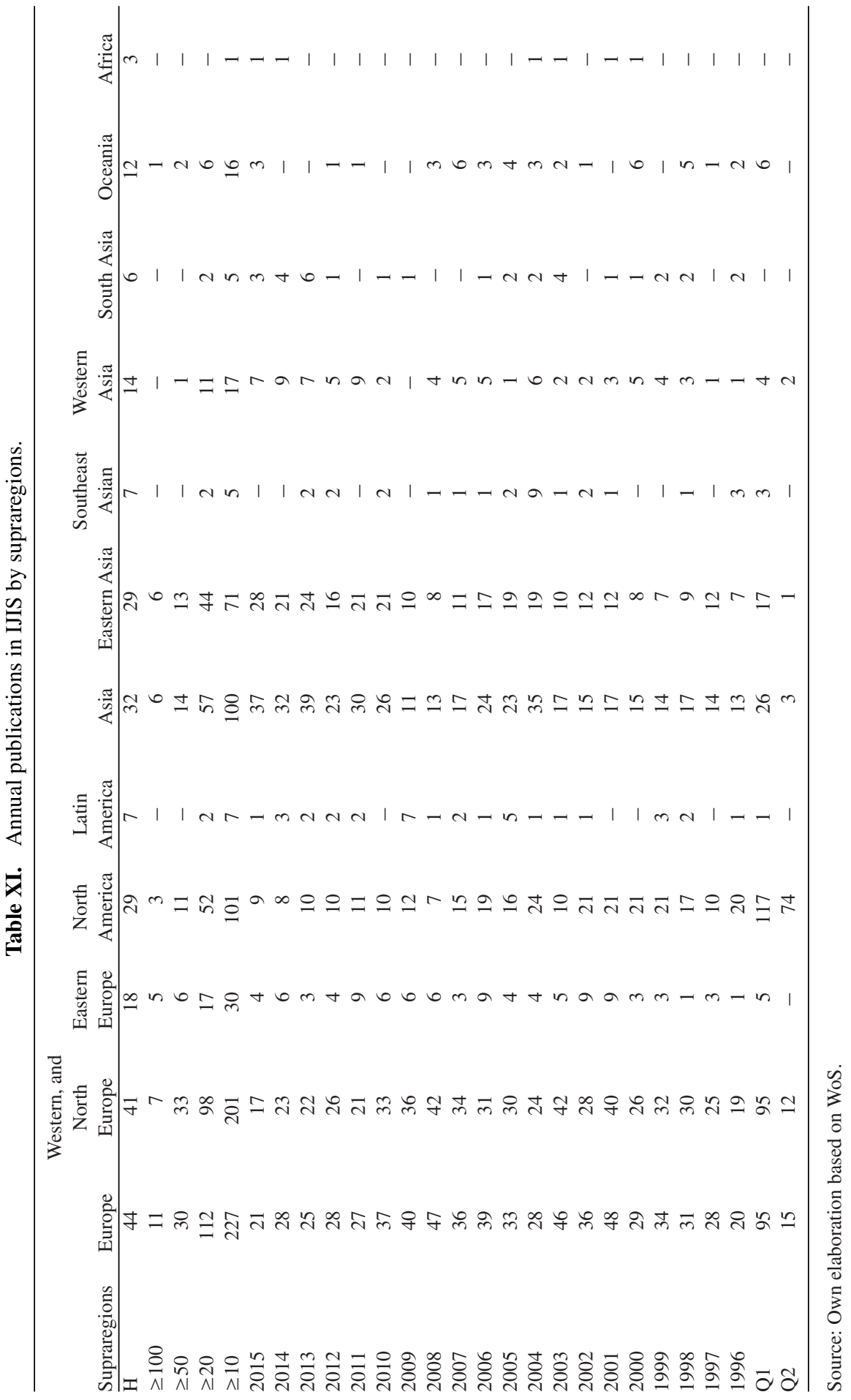




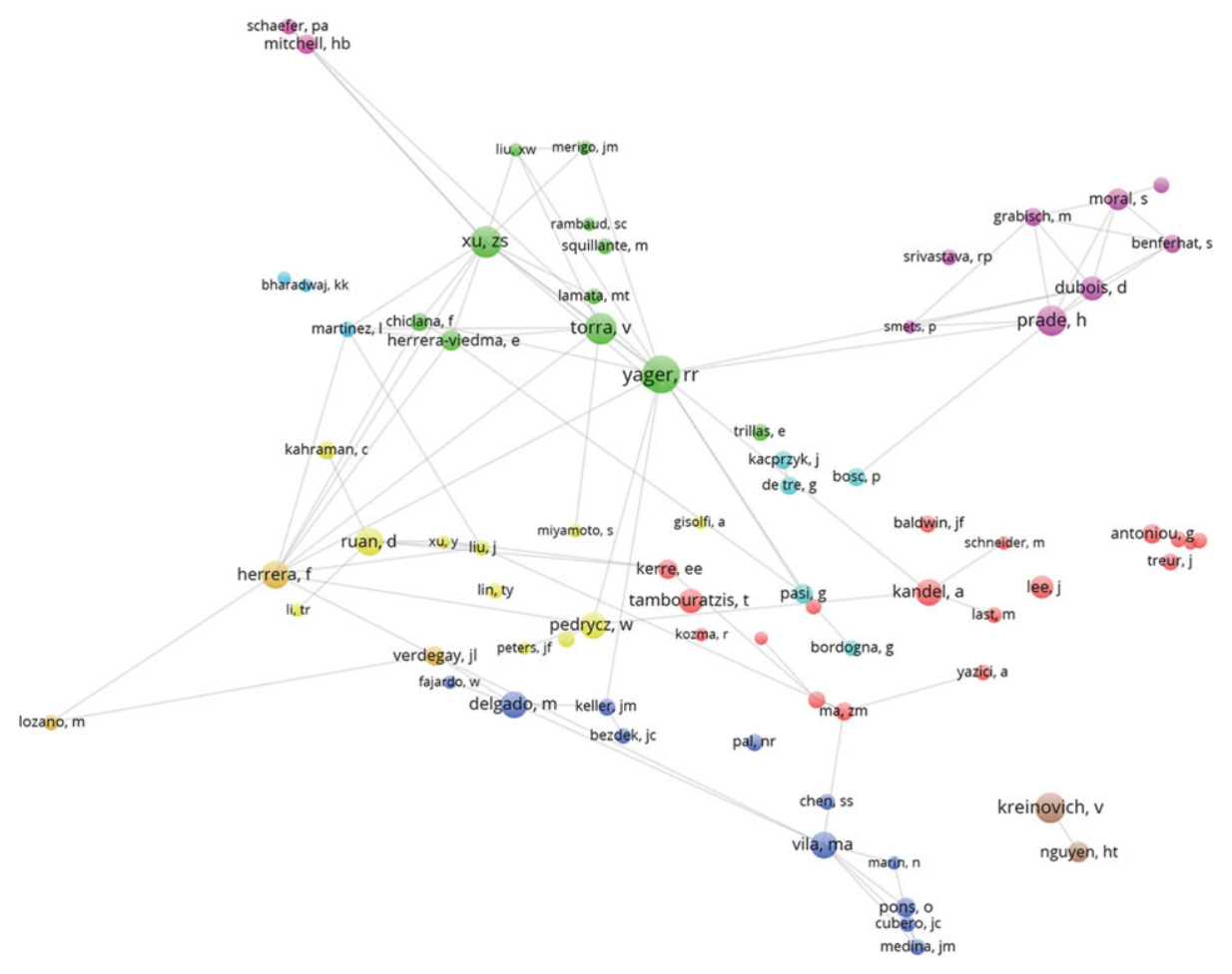

Figure 2. Bibliographic coupling of authors that publish in IJIS.

Now, let us focus on co-citation analysis. A first interesting issue here is to consider the most-cited papers in IJIS and to consider how they are connected with other papers. Figure 4 presents the co-citation of documents.

The most-cited paper in the journal is the seminal paper of Zadeh about "Fuzzy sets" that was published in 1965 in Information and Control. ${ }^{32}$ This paper is among the 50 most-cited papers of all time in all sciences and the most-cited paper in computer science. ${ }^{1}$ Very close to this paper appears the seminal paper of Yager about the OWA operator. ${ }^{33,34}$ Note also that several books are highly cited in the journal including the book of Shafer about the theory of evidence ${ }^{35}$ and the book of Klir and Yuan about fuzzy sets and systems. ${ }^{36}$ Zadeh and Yager are the authors with the highest number of papers in the graph.

A further interesting issue is to consider the co-citation of authors, that is, authors who have received the highest number of citations in the journal and how their profile is connected to other authors. Figure 5 presents the leading cocited authors of IJIS.

Yager is the most-cited author in journalism, closely followed by Zadeh. These two authors clearly represent the core of this journal. However, other authors also reach a significant position including Dubois, $\mathrm{Xu}$, Herrera, and Kacprzyk. With the exception of some highly cited authors, most of these authors appeared in Table IV through their books, including Glenn Shafer, Judea Pearl, and George J. Klir. 


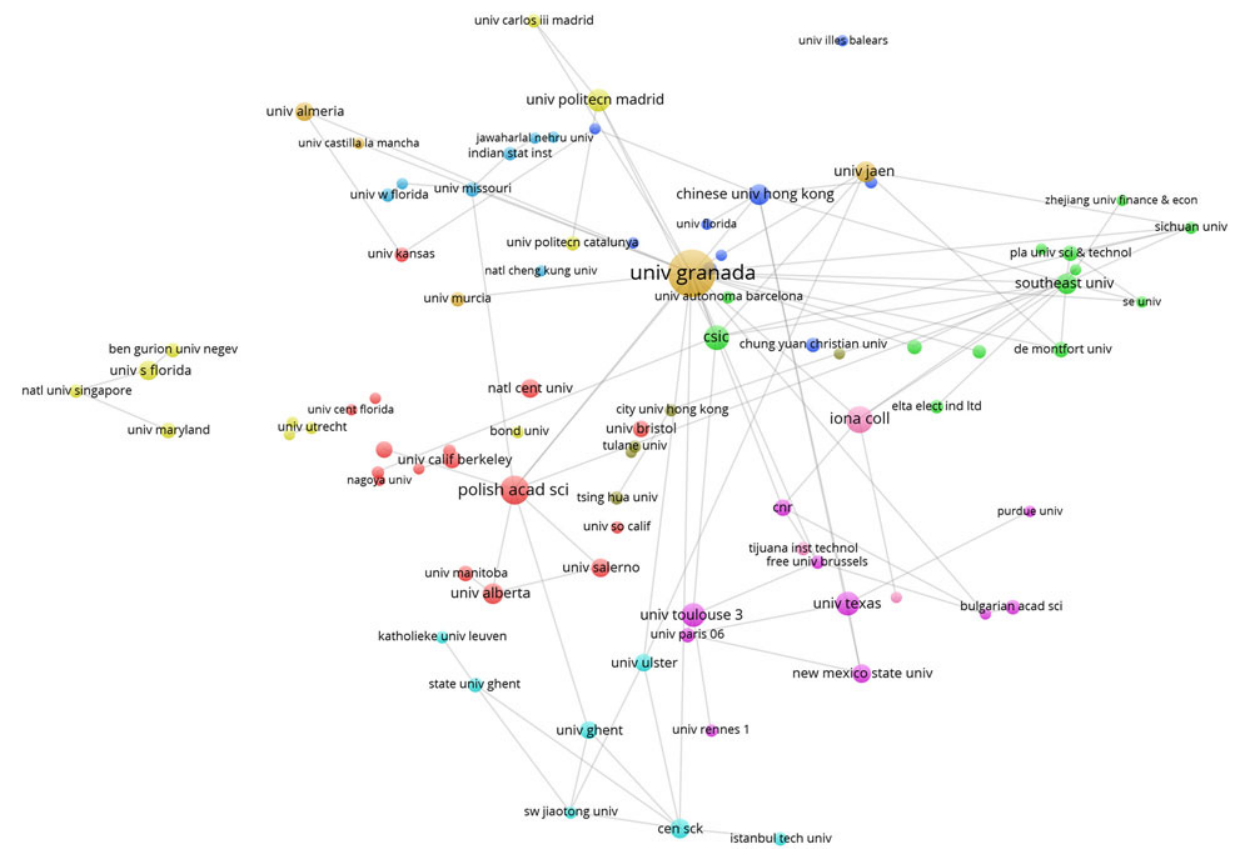

Figure 3. Bibliographic coupling of institutions that publish in IJIS.

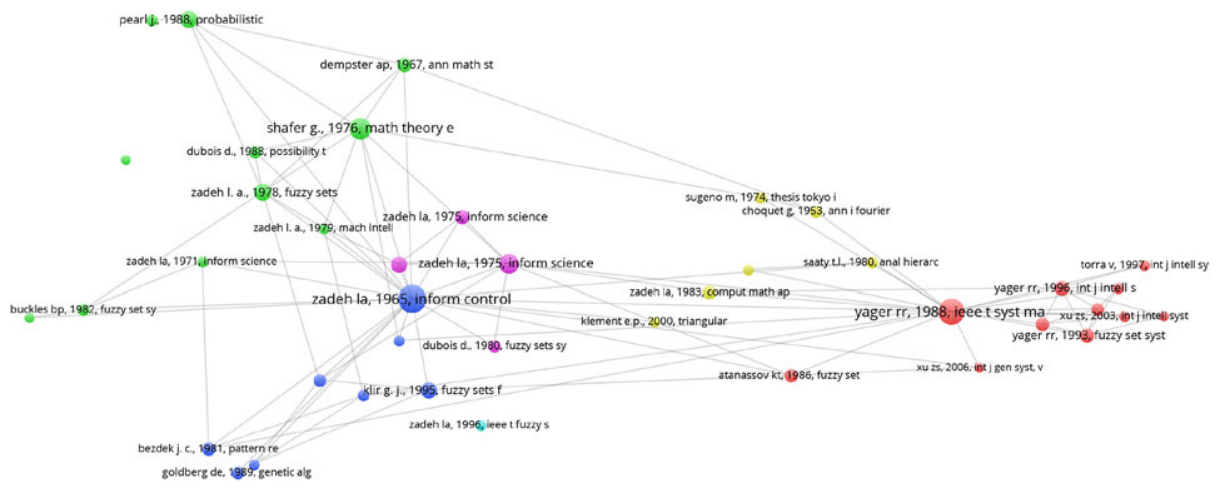

Figure 4. Co-citation of documents cited in IJIS.

Next, let us analyze the co-citation of journals in IJIS, that is, those journals that have received the highest number of citations in the journal. Figure 6 presents the results.

Fuzzy Sets and Systems is the most-cited journal in IJIS, followed by IJIS itself. Close to these appear Information Sciences, Artificial Intelligence, and the IEEE Transactions on Systems, Man and Cybernetics that now is divided into 


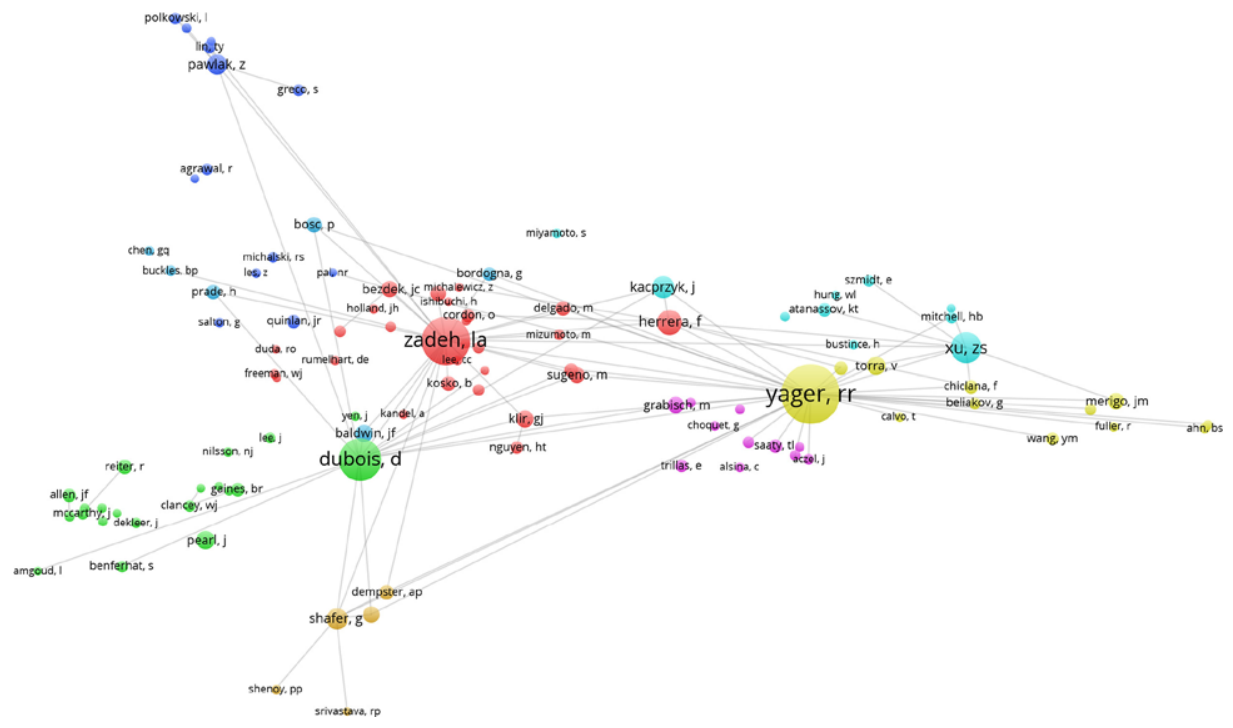

Figure 5. Co-citation of authors cited in the journal.

three journals. Most of the journals are from the computer science area, although other areas close to this field also appear including engineering, mathematics, and operations research.

Finally, let us develop a keyword analysis of the journal to identify the most frequently used keywords in the journal. Figure 7 presents the leading keywords of IJIS.

As seen, keywords such as preface, special issue, case, review, and so on are quite common in many journals. However, some groups of keywords clearly show the profile of the journal. First, at the bottom of the graph we see several keywords about decision making, aggregation operators, and OWA operators. At the rightbottom side of the graph, we identify several keywords about fuzzy sets and systems. These two groups are the most significant, although there are others in the fields of probability, knowledge, and, more generally, of computational intelligence.

\section{CONCLUSIONS}

The IJIS is now 30 years old. Many research contributions have been published during this time. This study has presented a bibliometric review of the journal during this period to identify the leading trends that have occurred in the journal. The analysis has been performed by searching for the bibliographic material in the WoS database. ${ }^{37}$ Several bibliometric indicators have been considered including the total number of publications and citations, the $h$-index, cites per paper, citation thresholds, and citing articles. The results indicate that IJIS is a leading journal in the computer science field, with a special focus on intelligent systems construction that includes 


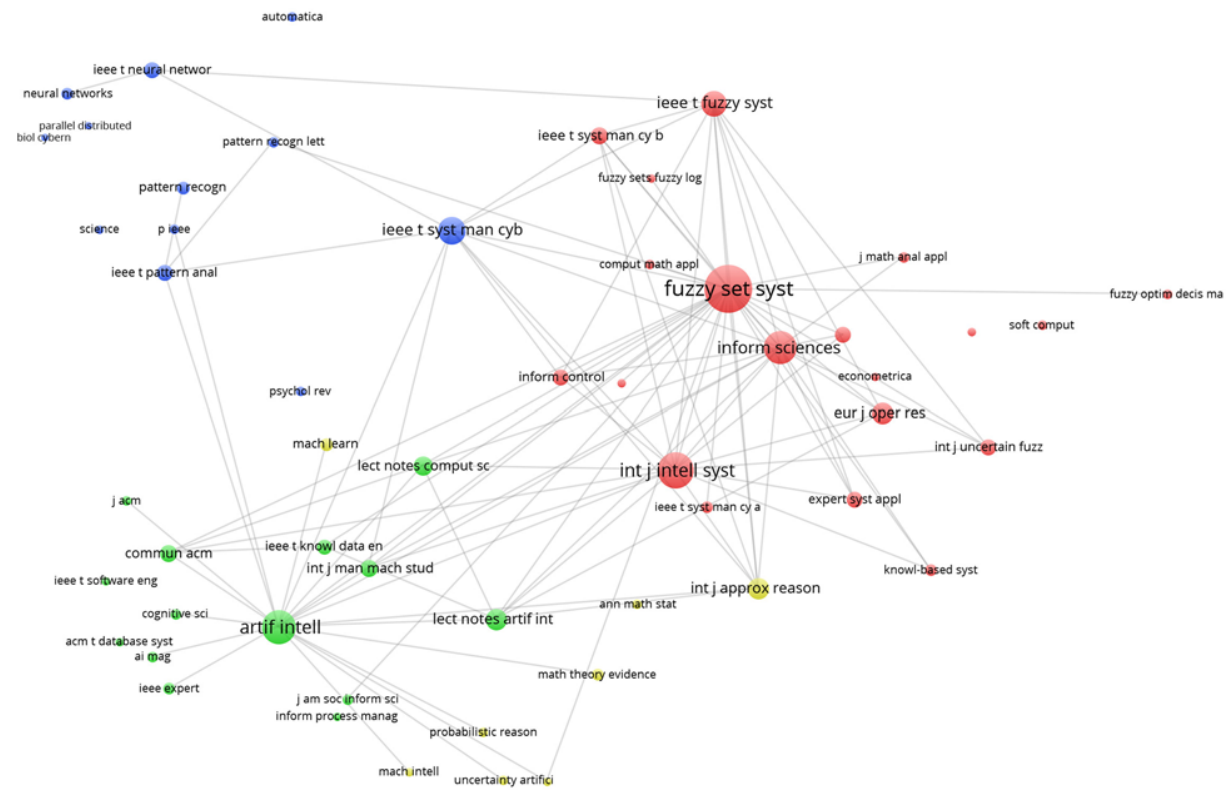

Figure 6. Co-citation of journals cited in IJIS.

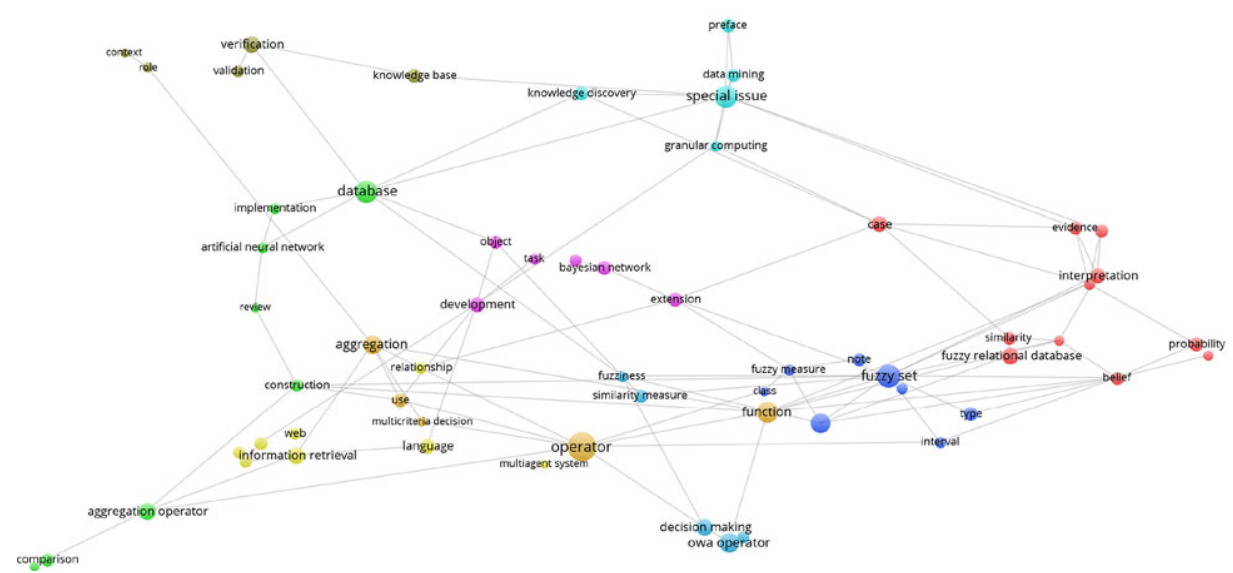

Figure 7. Keyword analysis of IJIS.

fuzzy systems, aggregation operators, decision making, and computational intelligence in general. The journal is strongly connected to other leading journals such as Fuzzy Sets and Systems, Information Sciences, IEEE Transactions on Systems, Man and Cybernetics (now divided into three parts), and the IEEE Transactions on Fuzzy Systems. Zeshui Xu and Ronald R. Yager are the most productive and influential authors in the journal in terms of papers published and citations that these papers have 
received. The University of Granada is the leading institution according to these indicators. From these results, three countries show the most significant presence in the journal: China, USA, and Spain.

The article also develops a graphical analysis of the publication and citation structure of the journal to map the leading issues in the journal and see how they are connected. For doing so, the paper has studied bibliographic coupling and cocitation. Bibliographic coupling shows the papers that are published in the journal and how they tend to cite the same material. Co-citation analyzes the material cited primarily in the journal and determines which published documents tend to receive citations from the same sources. The graphical results are quite consistent with the results seen in the tables. However, note that the most-cited authors in the journal are Lotfi A. Zadeh and Ronald R. Yager; their seminal papers are the leading documents on fuzzy sets ${ }^{32}$ and OWA operators. ${ }^{34,38}$

This paper provides an overview of the leading trends in the journal over the last 30 years. This approach is useful to obtain a general overview. However, note some limitations. First, the results can change over time, and many new issues might appear and condition the journal in the future. Second, we follow the methodology that WoS uses for classifying bibliographic material. However, this approach has limitations that also affect this study. ${ }^{39}$ For example, it provides one unit to any participating author, institution, and country in the paper. However, it could also be interesting to develop a fractional counting. Note that the work has implemented fractional counting in the VOS viewer analysis but not in the tables. In any case, the approach of WoS is acceptable because it is not easy to design a fractional counting because some authors might have contributed more to the paper and so on. Finally, it is worth mentioning that the article measures the bibliographic material. However, productivity and influence can often be conditioned by popularity and other related factors. Therefore, very good research might occasionally not appear highly rated. In this context, the paper aims to identify leading trends and be informative. However, many exceptional cases can appear in the analysis.

\section{Acknowledgment}

Support from the Chilean Government through the Fondecyt - Regular program (1160286) is gratefully acknowledged.

\section{References}

1. Merigó JM, Gil-Lafuente AM, Yager RR. An overview of fuzzy research with bibliometric indicators. Appl Soft Comp 2015;27:420-433.

2. Broadus RN. Toward a definition of "bibliometrics." Scientometrics 1987;12:373-379.

3. Coupé T. Revealed performances: worldwide rankings of economists and economics departments, 1990-2000. J Eur Econ Assoc 2003;1:1309-1345.

4. Podsakoff PM, MacKenzie SB, Podsakoff NP, Bachrach DG. Scholarly influence in the field of management: a bibliometric analysis of the determinants of university and author impact in the management literature in the past quarter century. J Manage 2008;34:641-720.

5. Cobo MJ, Martínez MA, Gutiérrez-Salcedo M, Fujita H, Herrera-Viedma E. 25 years at knowledge-based systems: a bibliometric analysis. Knowl-Based Syst 2015;80:3-13. 
6. Merigó JM, Mas-Tur A, Roig-Tierno N, Ribeiro-Soriano D. A bibliometric overview of the Journal of Business Research between 1973 and 2014. J Bus Res 2015;68:2645-2653.

7. Cakir MP, Acarturk C, Alasehir O, Cilingir C. A comparative analysis of global and national university ranking systems. Scientometrics 2015;103:813-848.

8. Bonilla C, Merigó JM, Torres-Abad C. Economics in Latin America: a bibliometric analysis. Scientometrics 2015;105:1239-1252.

9. Franceschini F, Maisano D. Sub-field normalization of the IEEE scientific journals based on their connection with technical societies. J Informetrics 2014;8:508-533.

10. Tsai CF. Citation impact analysis of top rated computer science journals and their rankings. J Informetrics 2014;8:318-328.

11. Godoy D, Zunino A, Mateos C. Publication practices in the Argentinean computer science community: a bibliometric perspective. Scientometrics 2015;2:1795-1814.

12. Singh VK, Uddin A, Pinto D. Computer science research: the top 100 institutions in India and in the world. Scientometrics 2015;104:529-553.

13. Uddin A, Singh VK, Pinto D, Olmos I. Scientometric mapping of computer science research in Mexico. Scientometrics 2015;105:97-114.

14. Van Eck NJ, Waltman L. Bibliometric mapping of the computational intelligence field. Int J Uncert Fuzz Knowl-Based Syst 2007;15:625-645.

15. $\mathrm{Yu} \mathrm{D}$. A scientometrics review on aggregation operator research. Scientometrics 2015;105:115-133.

16. Yu D, Shi SS. Researching the development of Atanassov intuitionistic fuzzy set: using a citation network analysis. Appl Soft Comput 2015;32:189-198.

17. Yu D, Li DF, Merigó JM, Fang L. Mapping development of linguistic decision making studies. J Intell Fuzzy Syst 2016;30:2727-2736.

18. Yin MS. Fifteen years of grey system theory research: a historical review and bibliometric analysis. Expert Syst Appl 2013;40:2767-2775.

19. Deng GF, Lin WT. Citation analysis and bibliometric approach for ant colony optimization from 1996 to 2010. Expert Syst Appl 2012;39:6229-6237.

20. Van Eck NJ, Waltman L. Software survey: VOSviewer, a computer program for bibliometric mapping. Scientometrics 2010;84:523-538.

21. Martyn J. Bibliographic coupling. J Doc 1964;20:236.

22. Small H. Co-citation in the scientific literature: a new measure of the relationship between two documents. J Am Soc Inform Sci 1973;24:265-269.

23. Alonso S, Cabrerizo FJ, Herrera-Viedma E, Herrera F. H-index: a review focused on its variants, computation, and standardization for different scientific fields. J Informetrics 2009;3:273-289.

24. Hirsch JE. An index to quantify an individual's scientific research output. Proc Natl Acad Sci U S A 2005;102:16569-16572.

25. Martínez MA, Herrera M, López-Gijón J, Herrera-Viedma E. H-classics: characterizing the concept of citation classics through H-index. Scientometrics 2014;98:1971-1983.

26. Yager RR. Quantifier guided aggregation using OWA operators. Int J Intell Syst 1996;11:49_ 73.

27. Xu ZS, Da QL. An overview of operators for aggregating information. Int J Intell Syst 2003;18:953-969.

28. $\mathrm{Xu} \mathrm{ZS}$. An overview of methods for determining OWA weights, Int $\mathrm{J}$ Intell Syst 2005;20:843-865.

29. Torra V. The weighted OWA operator. Int J Intell Syst 1997;12:153-166.

30. Torra V. Hesitant fuzzy sets. Int J Intell Syst 2010;25:529-539.

31. Merigó JM, Cancino C, Coronado F, Urbano D. Academic research in innovation: a country analysis. Scientometrics 2016;108:559-593.

32. Zadeh LA. Fuzzy sets. Inform Control 1965;8:338-353.

33. Emrouznejad A, Marra M. Ordered weighted averaging operators 1988-2014. A citation based literature survey. Int J Intell Syst 2014;29:994-1014.

34. Yager RR. On ordered weighted averaging aggregation operators in multi-criteria decision making. IEEE Trans Syst Man Cybern B 1988;18:183-190. 
35. Shafer G. Mathematical theory of evidence. Princeton, NJ: Princeton University Press; 1976.

36. Klir GJ, Yuan B. Fuzzy sets and fuzzy logic: Theory and applications. Upper Saddle River, NJ: Prentice Hall; 1995.

37. Merigó JM, Rocafort A, Aznar-Alarcón JP. A bibliometric overview of business \& economics research. J Bus Econ Manag 2016;17:397-413.

38. Yager RR, Kacprzyk J, Beliakov G. Recent developments on the ordered weighted averaging operators: Theory and practice. Berlin: Springer-Verlag; 2011.

39. Merigó JM, Núñez A. Influential journals in health research: a bibliometric study. Globalization Health 2016;12:46. 